\title{
DFT $+U$ study of self-trapping, trapping, and mobility of oxygen-type hole polarons in barium stannate
}

\author{
Grégory Geneste, Bernard Amadon, and Marc Torrent \\ CEA, DAM, DIF, F-91297 Arpajon, France
}

\author{
Guilhem Dezanneau \\ Laboratoire Structures, Propriétés et Modélisation des Solides, CentraleSupelec, CNRS, UMR8580, Université Paris-Saclay, \\ 8 rue Joliot Curie 91190 Gif sur Yvette, France
}

(Received 16 December 2016; revised manuscript received 26 September 2017; published 30 October 2017)

\begin{abstract}
The charge-transfer insulating perovskite oxides currently used as fuel cell electrolytes undergo, at high temperature, an oxidation reaction $\frac{1}{2} \mathrm{O}_{2}(\mathrm{~g})+\mathrm{V}_{\mathrm{O}}^{\bullet \bullet} \rightarrow \mathrm{O}_{\mathrm{O}}^{\mathrm{X}}+2 \mathrm{~h}^{\bullet}$, that produces oxygen-type holes. Understanding the nature and mobility of these oxygen-type holes is an important step to improve the performance of devices, but presents a theoretical challenge since, in their localized form, they cannot be captured by standard density functional theory. Here, we employ the DFT $+U$ formalism with a Hubbard correction on the $p$ orbitals of oxygen to investigate several properties of these holes, in the particular case of $\mathrm{BaSnO}_{3}$. We describe the small oxygen-type hole polarons, the self-trapping at their origin, and their trapping by trivalent dopants ( $\mathrm{Ga}, \mathrm{Sc}, \mathrm{In}, \mathrm{Lu}$, Y, Gd, La). Strong similarities with protonic defects are observed concerning the evolution of the trapping energy with ionic radius of the dopant. Moreover, we show that long-range diffusion of holes is a complex phenomenon, that proceeds by a succession of several mechanisms. However, the standard implementation of DFT $+U$ within the projector augmented-wave (PAW) formalism leads to use very large, unphysical values of $U$ for the O- $p$ orbital. We propose here a slightly modified DFT $+U$ scheme, that takes into account the fact that the O- $p$ is truncated in usual DFT $+U$ implementation in PAW. This scheme yields more physical values of $U$ than the ones traditionally used in the literature, and describes well the properties of the hole polaron.
\end{abstract}

DOI: 10.1103/PhysRevB.96.134123

\section{INTRODUCTION}

Solid oxide fuel cells (SOFCs) and protonic ceramic fuel cells (PCFCs) are promising devices for the conversion into electricity of the chemical energy stored in the $\mathrm{H}_{2}$ molecule. They could overcome several drawbacks encountered in polymer exchange membrane fuel cells. For instance, these devices work at sufficiently high temperature to allow easy dissociation of the dihydrogen molecule and avoid the use of expensive catalysts such as platinum. The electrolytes of SOFCs and PCFCs are oxide compounds in which an aliovalent doping (substitution of cations by elements of lower valence) creates, by charge compensation, a large concentration of charged oxygen vacancies $\mathrm{V}_{\mathrm{O}}^{\bullet \bullet}$. In $\mathrm{SOFCs,}$ these charged oxygen vacancies allow oxide ion migration, while in PCFCs, they are favorable to water dissociation when put in contact with humid atmosphere. This creates ionic conductivity, by the migration of $\mathrm{O}^{2-}$ ions in the first case, and protonic conductivity in the latter [1]. In PCFCs, the materials currently used as electrolytes are insulating perovskite oxides such as $\mathrm{BaZrO}_{3}$, $\mathrm{BaSnO}_{3}$, or $\mathrm{BaCeO}_{3}$, doped by trivalent elements on their $\mathrm{B}$ site.

However, at the high working temperatures of the devices (typically 600-1000 K), these compounds are subject to an oxidation reaction that may deteriorate their quality as electrolyte materials because it makes the compounds hole-type electronic conductors, whereas they should remain electronic insulators whatever the conditions. It consists in the dissociative insertion of oxygen molecules in the charged vacancies, according to

$$
\frac{1}{2} \mathrm{O}_{2}(\mathrm{~g})+\mathrm{V}_{\mathrm{O}}^{\bullet \bullet} \rightarrow \mathrm{O}_{\mathrm{O}}^{\mathrm{X}}+2 \mathrm{~h}^{\bullet}
$$

Since the zirconate, stannate, cerate, or titanate perovskites in question, with a tetravalent B ion, are charge-transfer insulators, the holes, that correspond to empty states at the top of the valence band, are formed by depopulated $2 p$ orbitals of oxygen. It has been suggested that they may be rather delocalized under the form of large polarons [2]. It is now commonly admitted that, in many charge-transfer oxides, these holes localize on single oxygen atoms, under the form of small polarons $\mathrm{O}^{-}$(that can be noted as $\mathrm{O}_{\mathrm{O}}^{\bullet}$ ) [3-7], which may be considered as true at least at low temperature. This oxygen-type self-trapped hole polaron (STHP) occupies, in these perovskite oxides, a $2 p$ orbital oriented perpendicular to the B-O-B bond $[4,8]$. Recent hybrid density functional calculations report self-trapping energies favorable to the formation of small oxygen-type hole polarons in $\mathrm{BaCeO}_{3}(-0.15 \mathrm{eV}$ [9]), in $\mathrm{SrTiO}_{3}\left(-0.05 \mathrm{eV}\right.$ [8]), and in $\mathrm{BaZrO}_{3}(-0.2 \mathrm{eV}$ [4]).

Improving the quality of the electrolytes in the hightemperature working regime of SOFCs/PCFCs requires to understand clearly this oxidation, including the behavior of the holes resulting from this reaction. Theoretically, previous studies have revealed that advanced electronic structure calculations are needed to correctly describe this oxidation phenomenon. Indeed, the generalized gradient approximation (GGA) finds it exothermic in several perovskite oxides [10-12], while hybrid functionals (PBE0) provide an endothermic picture [12]. This is related to the band-gap problem, more precisely to the incorrect position of the valence band maximum, formed by oxygen- $2 p$ states, obtained with GGA. Moreover, density functional theory (DFT) at its local and semilocal level of approximation (LDA, GGA) is unable to capture the localized oxygen-type hole polarons, while hybrid 
functionals such as PBE0 or HSE06 are able to $[13,14]$. This is mainly due to the self-interaction error that local and semilocal functionals exhibit. Alternatively, the so-called DFT $+U$ with an onsite correction Hubbard term $U$ applied on the $p$ orbitals of oxygen, may successfully remove part of this self-interaction [15]. Indeed, it has been used in the past years to describe oxygen-type hole polarons in various charge-transfer insulating oxides, including titanate/zirconate perovskite oxides $[4,13]$ or $\mathrm{TiO}_{2}[16,17]$. It is interesting to note that very large values of $U$ (typically $\sim 5-10 \mathrm{eV}$ ) are needed, typical of the ones used in strongly correlated systems. For instance, note the following:

(i) In titanates $A_{\mathrm{TiO}}(A=\mathrm{Pb}, \mathrm{Ba}, \mathrm{Sr})$, Erhart et al. [13] have shown that $\mathrm{LDA}+U$, with $U=8 \mathrm{eV}$, can be used to simulate oxygen-type self-trapped small hole polarons, reproducing in a satisfactory manner the results obtained on these entities by the hybrid HSE06 functional.

(ii) Very recently, Lindman et al. used DFT $+U$ to model the oxygen-type self-trapped hole polaron in $\mathrm{BaZrO}_{3}$ [4]: they predict a favorable self-trapping energy of $\sim-0.11 \mathrm{eV}$ $(U=6.5 \mathrm{eV})$, close to that provided by HSE06 on that system $(-0.2 \mathrm{eV})$, and a rather low activation energy for hole mobility $(\sim 0.2 \mathrm{eV})$.

The ability of DFT $+U$ to offer the same precision as hybrid functionals on such polaronic systems is thus well established. Besides, the lower computational cost of $\mathrm{DFT}+U$ enables to extend the study of self-trapped small hole polarons to larger and more complex systems. However, as pointed out by Lany and Zunger [18], the large value of $U$ that has to be introduced (necessary to describe well the localization effect of the polaron) may be inappropriate to describe simultaneously some properties of the host matrix.

In this work, we use DFT $+U$, with $U$ applied on the $p$ states of oxygen, to describe the self-trapped state, the trapping effect of dopants and the mobility of holes, in the case of barium stannate, $\mathrm{BaSnO}_{3}$ (BSO), a potential electrolyte material for SOFCs and PCFCs [19-28], for which hole-type conductivity at high temperature has been reported [20,27,28]. Besides, we propose a slightly modified DFT $+U$ scheme, more adapted to $p$ orbitals, and show that such scheme is able to provide similar physics, but with more physical values of $U$ than the ones traditionally used in the literature. This modified scheme, which is a simple renormalization of the number of electrons, will be denoted as scheme $\mathrm{o}_{\mathrm{C}}$ throughout the paper, while the standard one will be denoted as scheme $\mathrm{o}_{\mathrm{A}}$ (see Sec. II B and Appendix C).

$\mathrm{BSO}$, interestingly, also presents high level of electronic conductivity under $n$-type doping, which has been recently investigated using hybrid density functional approaches $[29,30]$. This last point, however, will not be treated in this work, and we only focus on holes, i.e., on the material behavior in oxidative atmosphere.

The paper is organized as follows. Section II gives computational details and presents this slightly modified $\mathrm{DFT}+U$ scheme. Then, we describe the oxygen-type hole polaron $\mathrm{O}^{-}$ in $\mathrm{BaSnO}_{3}$, as obtained by $\mathrm{GGA}+U$, either self-trapped in the lattice, or trapped in the vicinity of trivalent dopants $(\mathrm{Ga}, \mathrm{Sc}$, In, Lu, Y, Gd, La) (Sec. III). After that, we carefully investigate its mobility and show that hole diffusion occurs by a complex succession of hoppings and reorientations (Sec. IV).

\section{COMPUTATIONAL DETAILS}

\section{A. Density functional theory calculations}

Density functional theory calculations [31] have been performed using the ABINIT code [32]. The projector augmentedwave (PAW) formalism [33] is employed, with the generalized gradient approximation (GGA-PBE) [34] and the GGA-PBE $+U$, the Hubbard correction being applied on the $p$ states of oxygen (in what follows, the term $U$ denotes exclusively the Hubbard correction applied on the $p$ states of oxygen). The plane-wave cutoff is 25 Hartrees (50 Ha for the double grid). We use a $3 \times 3 \times 3$ supercell with fixed dimension (three times that of perfect $\mathrm{BaSnO}_{3}$ ), in terms of the primitive 5-atom unit cell (135 atoms), in which several defects are introduced in different charge states: (i) hole (i.e., electron removed, either localized or delocalized), (ii) trivalent dopant $(\mathrm{Ga}, \mathrm{Sc}$, In, Lu, Y, Gd, La) in charge state -1 and 0 (in the latter case, this corresponds to the association of a negative dopant and a hole). Two kinds of calculations are performed: structural optimizations and minimum energy paths.

Charged supercells are computed using a uniform compensating background, and the total energies are corrected according to the Makov-Payne scheme [35] (except in the case of the delocalized hole) and by a band alignment based on the $5 s$ semicore levels of barium [36]. The Brillouin zone of the supercell is sampled by a $2 \times 2 \times 2 k$-point mesh (increased to $4 \times 4 \times 4$ in the case of the delocalized hole, which is a metallic system). The various configurations are optimized until all the Cartesian components of the atomic forces are below $2.0 \times 10^{-4} \mathrm{Ha} / \operatorname{Bohr}(\sim 0.01 \mathrm{eV} / \AA)$. All the calculations involving a hole, either localized or delocalized, are spin polarized. Our $k$-point grid is shifted in the usual way. However, we have checked in particular cases that inclusion of the $\Gamma$ point does not modify the results: within scheme $o_{C}$, the total energy of the supercell containing a self-trapped hole polaron is changed by less than $1 \mathrm{meV}$ when using a nonshifted $2 \times 2 \times 2$ grid, while that of the supercell containing the delocalized hole is changed by less than $5 \mathrm{meV}$ when using a nonshifted $4 \times 4 \times 4$ grid.

The PAW atomic data sets are described in Appendix A (PAW radii, semicore electrons). In the ABINIT and VASP codes, the DFT $+U$ is implemented on the PAW spheres only (see discussion in the next subsection). Thus, the radius of the PAW atomic data set is likely to have a large influence on the DFT $+U$ results, especially in the case of the $2 p$ orbitals of $\mathrm{O}$, which are rather extended in space. Here, an oxygen atomic data set with a radius of 1.6 a.u. has been used in all the DFT $+U$ calculations (except in Appendix D, where an atomic data set with a radius of 1.4 a.u. has been tested). The PBE calculations, provided here for comparison, have been performed using a 1.4 a.u. data set. For Sn, a PAW atomic data set treating explicitly the $4 d$ electrons has been used for all the calculations, except for the computation of the minimum energy paths for hopping/reorientation of the hole polarons, where the $4 d$ electrons of Sn are frozen in the core of the atomic data set (the plane-wave cutoff is $18 \mathrm{Ha}$ in that case). The energy barriers for hole migration have been computed using the simplified string method [37]. 


\section{B. DFT $+U$ peculiarities}

Formally, DFT $+U$ formalism could be applied to any kind of correlated orbitals. However, and as discussed by Lany and Zunger [18], implementations of DFT $+U$ in PAW lead to a huge variations of the number of electrons for O- $p$ orbitals, depending on the choice of PAW atomic data. In this section, we discuss a simple modification that has to be done in the PAW implementation of the DFT $+U$ in order that calculations on O- $p$ orbitals are of the same precision as calculations on more localized orbitals $(d$ or $f$ ).

The main physical quantity which occurs in a DFT $+U$ calculation is the density matrix of correlated orbitals $n_{m, m^{\prime}}^{l, \sigma}$ [38]. The diagonal element of this matrix is the number of electrons in the orbital indexed by quantum numbers $l$ and $m$ and spin $\sigma$. This density matrix can be written simply as

$$
n_{m, m^{\prime}}^{l, \sigma}=\sum_{\mathbf{k}, v} f_{v}^{\mathbf{k}, \sigma}\left\langle\Psi_{v}^{\mathbf{k}, \sigma} \mid \chi_{l m^{\prime}}\right\rangle\left\langle\chi_{l m} \mid \Psi_{v}^{\mathbf{k}, \sigma}\right\rangle
$$

where $\Psi_{v}^{\mathbf{k}, \sigma}$ and $f_{v}^{\mathbf{k}, \sigma}$ are the Kohn-Sham orbital and its occupation for $k$ point $\mathbf{k}$, spin $\sigma$, and band $v$. Importantly, the density matrix also depends on the definition of correlated orbitals $\chi_{l m}$. The angular part of $\chi_{l m}$ is the spherical harmonic $Y_{l m}$ and is well defined by the angular momentum (here $l=1$ for $p$ orbitals). However, the radial part of $\chi_{l m}$ is more subject to variation. This radial part can be the radial part of LDA (or LDA $+U$ ) atomic orbitals, of truncated orbitals [39,40], or of Wannier functions [41]. The DFT $+U$ formulation $[39,40]$ often used in the projector augmentedwave (PAW) method (scheme $\mathrm{o}_{\mathrm{A}}$, as in ABINIT and VASP [see Eq. (C1) in Appendix C]) is in practice equivalent [40] to using renormalized truncated atomic orbitals $\bar{\phi}_{0}^{t}$ as the radial part of correlated orbitals [scheme $\mathrm{o}_{\mathrm{B}}$, as in Eq. (C2) in Appendix C]. Renormalized truncated atomic orbitals are used instead of atomic orbitals because in order to simplify the PAW implementation, the projection of atomic orbitals over Kohn-Sham orbitals $\left(\left\langle\Psi_{v}^{\mathbf{k}, \sigma} \mid Y_{l m} \bar{\phi}_{0}^{t}\right\rangle\right)$ is only done inside the PAW atomic sphere defined by the PAW radius [40]. So, the renormalization is here to compensate for the part of the correlated orbital which is outside the PAW atomic sphere. For the sake of completeness, Fig. 1 compares radial parts of an atomic orbital $\phi_{0}$, a truncated atomic orbital $\phi_{0}^{t}$, and a renormalized atomic orbital $\bar{\phi}_{0}^{t}=\frac{\phi_{0}^{t}}{\sqrt{\left\langle\phi_{0}^{t} \mid \phi_{0}^{t}\right\rangle}}$ for an oxygen- $p$ orbital.

Let us now investigate the consequence of this truncation on the scalar product $\left\langle\Psi_{v}^{\mathbf{k}, \sigma} \mid Y_{l m} \bar{\phi}_{0}^{t}\right\rangle$. We focus on the particular case of an oxygen atom and we select the Kohn-Sham wave function of a $p$ orbital which can be written as $\Psi=Y_{\underline{l m}} \phi_{0}$. In this case, this scalar product is simply $\left\langle\phi_{0} \mid \bar{\phi}_{0}^{t}\right\rangle=\left\langle\phi_{0}^{t} \mid \bar{\phi}_{0}^{t}\right\rangle=$ $\sqrt{\left\langle\phi_{0}^{t} \mid \phi_{0}^{t}\right\rangle}$. Indeed, not only the atomic orbital, but also the Kohn-Sham orbital is truncated because of the scalar product $\left\langle\Psi_{v}^{\mathbf{k}, \sigma} \mid Y_{l m} \bar{\phi}_{0}^{t}\right\rangle$ which appears in Eq. (C2). Thus, using Eq. (C2), and as can be seen on Table $\mathrm{I}$, the maximal number of $p$ electrons within this scheme is not 6 as it should be, but $6\left\langle\phi_{0}^{t} \mid \phi_{0}^{t}\right\rangle$ (the precise value depends on the PAW radius): it is thus a drawback of the truncation of the scalar product as it is commonly done in PAW. This drawback has limited consequences for $d$ and $f$ orbitals which are mainly localized inside the PAW sphere. However, for the $p$ orbital, the

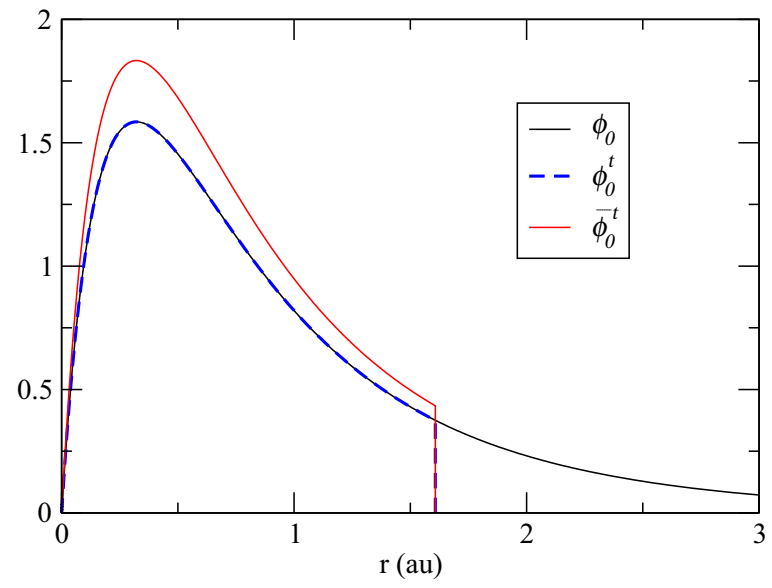

FIG. 1. Comparison of the radial part of an atomic orbital $\phi_{0}$, a truncated atomic orbital $\phi_{0}^{t}$, and a renormalized atomic orbital $\bar{\phi}_{0}^{t}$ for an oxygen- $p$ orbital.

problem is more important and the number of electrons cannot be typically greater than 4.5. A solution to this problem would be to implement the calculation of the density matrix without truncation of the scalar product. It is, however, an important task beyond the scope of this work because it requires an important modification of the DFT $+U$ Kohn-Sham potential, of the total energy and of the expression of forces on atoms. Here, we focus on a very simple way to circumvent this problem. One can easily see that the use of a correct renormalization of the density matrix as in Eq. (C4) (scheme $\mathrm{o}_{\mathrm{C}}$ ) enables to recover the good number of electrons (see Appendix $\mathrm{C}$ for an extensive discussion). This renormalization is equivalent to renormalize the scalar product $\left\langle\Psi_{v}^{\mathbf{k}, \sigma} \mid Y_{l m} \bar{\phi}_{0}^{t}\right\rangle$ with $\sqrt{\left\langle\phi_{0}^{t} \mid \phi_{0}^{t}\right\rangle}$ in order that the scalar product is simply one in the atomic limit. We emphasize that this modification does not modify the physical meaning of the DFT $+U$ approximation and is just a simple renormalization of the scalar product in order to recover the correct number of electrons and thus the correct physics (see Table I).

As we emphasize below, for an oxygen with a hole polaron in $\mathrm{BaSnO}_{3}$, this simple change in the calculation of the density matrix enables to obtain a more physical value of $U$ (see next section), and also more physical values for the number of electrons and the band gap.

TABLE I. Comparison of the maximal number of electrons of oxygen- $p$ orbital in the standard projection scheme of DFT $+U\left(\mathrm{o}_{\mathrm{A}}\right.$ or $\mathrm{o}_{\mathrm{B}}$ ), as used in ABINIT and VASP, and using our renormalized projection $\left(\mathrm{o}_{\mathrm{C}}\right)$. The numerical results for $\mathrm{o}_{\mathrm{B}}$ depend on the value of the PAW radius. For a typical value, standard projection severely underestimates the number of electrons, but our renormalized projection recovers the correct result. For $d$ and $f$ orbitals which are mainly inside the PAW sphere, the two schemes are close to each other.

\begin{tabular}{lc}
\hline \hline Occupation matrix & Max. number of electrons \\
\hline Standard projection $\left(\mathrm{o}_{\mathrm{A}}\right)$ & $4.44=6\left\langle\phi_{0}^{t} \mid \phi_{0}^{t}\right\rangle$ \\
Renormalized projection $\left(\mathrm{o}_{\mathrm{C}}\right)$ & 6.00 \\
\hline \hline
\end{tabular}



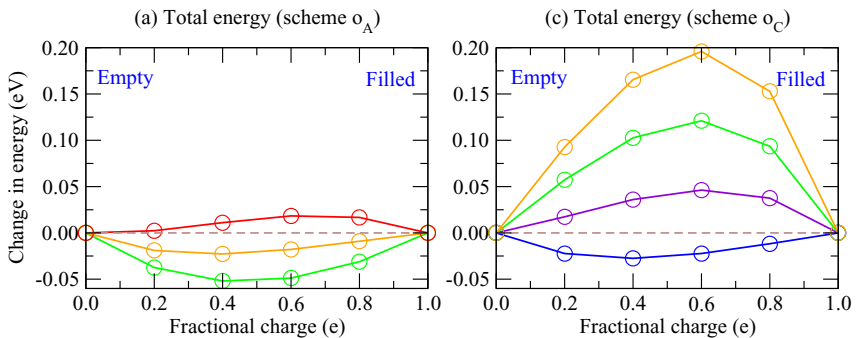

(b) $\mathrm{DFT}+\mathrm{U}$ contribution $\left(\right.$ scheme $\mathrm{o}_{\mathrm{A}}$ )

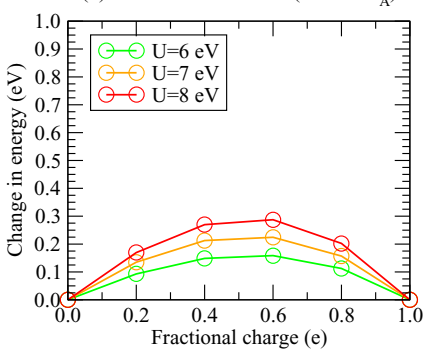

(d) $\mathrm{DFT}+\mathrm{U}$ contribution (scheme $\mathrm{o}_{\mathrm{C}}$ )

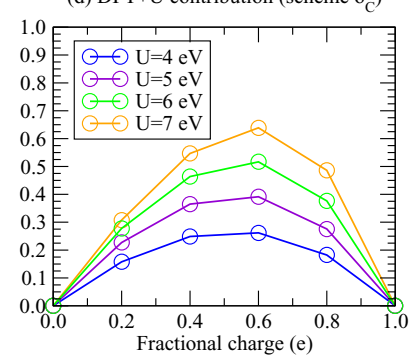

FIG. 2. Energy change with respect to piecewise linearity, as a function of fractional charge (number of electrons) in the polaron, and DFT $+U$ contribution to the total energy, due to the orbital on which the hole is located, namely, $U / 2\left(n_{i}-n_{i}^{2}\right)$. The linearity is subtracted so that the concavity or convexity appears clearly. Left panels: standard DFT $+U$ scheme $\left(\mathrm{o}_{\mathrm{A}}\right)$, with $U=6,7$, and $8 \mathrm{eV}$; right panels: scheme $\mathrm{o}_{\mathrm{C}}(U=4,5,6$, and $7 \mathrm{eV})$. The atomic geometry (positions, lattice constant) is fixed to that of the STHP at $U=6 \mathrm{eV}$ (with scheme $o_{A}$ ).

\section{Piecewise linearity of the energy as a function of fractional charge}

We now determine which value of $U$ must be used to simulate the oxygen-type hole polarons in BSO. In a selftrapped hole polaron, the hole is localized in space and thus is no more in a Bloch state. As a consequence, it exhibits self-interaction effects [42] when calculated in GGA-PBE, which destabilize the polaron and favor the delocalized state. Following Erhart et al. [13] and Lindman et al. [4], we compute, in the STHP geometry, the energy as a function of a fractional excess electronic charge in PBE $+U$. We plot this energy on Fig. 2 for both DFT $+U$ schemes, as a function of excess charge and for different values of $U$.

In such situation, it is known that deviations from the piecewise linearity are due to the self-interaction error of GGA $[15,18]$. Whereas LDA and PBE exhibit convexity, the Hubbard correction is concave and can thus restore the piecewise linearity [15] of energy. The same correction can be done by a contribution of exact exchange as in hybrid functionals [13].

Using scheme $\mathrm{o}_{\mathrm{A}}$, the energy is convex for $U=6$ and $7 \mathrm{eV}$, whereas for $U=8 \mathrm{eV}$, the energy is concave. The value of $U$ for which the self-interaction disappears is thus $\simeq 7.5 \mathrm{eV}$ within this DFT $+U$ scheme. We emphasize that such value is, however, dependent on the radius of the PAW atomic data set, here equal to 1.6 a.u.

We now perform the same calculations using scheme $o_{C}$ [Eq. (C4)]. The results are plotted on Fig. 2 (right panels) for $U=4,5,6$, and $7 \mathrm{eV}$ (with the linearity subtracted), allowing comparison with the standard implementation (scheme $\mathrm{o}_{\mathrm{A}}$ ).
We see that the optimal value of $U$ is dependent on the choice of the density matrix. Whereas the optimal $U$ is $\sim 7.5 \mathrm{eV}$ in scheme $\mathrm{o}_{\mathrm{A}}$ [Eq. (C1)], it is much smaller, $\sim 4.5 \mathrm{eV}$, in scheme $\mathrm{o}_{\mathrm{C}}$ [Eq. (C4)]. It can be interpreted easily: as discussed above, the values of the number of electrons in each orbital are larger in scheme $o_{C}[\mathrm{Eq} .(\mathrm{C} 4)]$ with respect to scheme $\mathrm{o}_{\mathrm{A}}$ [Eq. (C1)]. Let us call $n_{i}$ the number of electrons in the orbital $i$, for excess charge $q$. The DFT $+U$ correction to concavity $\left[\frac{U}{2} \sum_{i}\left(n_{i}-n_{i}^{2}\right)\right]$ is different in the two schemes. ${ }^{1}$ Moreover, as $n_{i}=n_{i}^{0}$ is lower than 1 (whatever the scheme, because of hybridization effects), when the hole is completely filled ( $q=0$, number of electrons $=1$ on Fig. 2 ), there is a linear contribution in the DFT $+U$ correction which is $\frac{U}{2} \sum_{i}(1-$ $\left.n_{i}^{0}\right) n_{i}$, thus, the deviation to linearity of the DFT $+U$ correction is $\frac{U}{2} \sum_{i} n_{i}\left(n_{i}^{0}-n_{i}\right)$. In this quantity, the main effect comes from the oxygen- $p$ orbital in which the hole is located. So, we plot this quantity for this orbital only (and for the two schemes) on Fig. 2 (bottom panels). We see that the DFT $+U$ correction is much lower for $\mathrm{o}_{\mathrm{A}}$ [Eq. $\left.(\mathrm{C} 1)\right]$ with respect to $\mathrm{o}_{\mathrm{C}}$ [Eq. (C4)] because $n_{i}^{0}$ is lower in the former case (coherently with the data in Table V). As a consequence, it explains why a lower $U$ is required in the scheme o $[\mathrm{Eq}$. (C4)] to achieve linearity of the total energy, and cancellation of the GGA-PBE self-interaction error. As we emphasize in Appendix C, using the scheme $o_{C}[\mathrm{Eq} .(\mathrm{C} 4)]$ is much more physical. We stress that a fair comparison between different calculations requires to use not only the same scheme $\left(\mathrm{o}_{\mathrm{A}}\right.$ or $\left.\mathrm{O}_{\mathrm{C}}\right)$, but also the same $P A W$ radius for oxygen atom.

\section{OXYGEN-TYPE HOLE POLARON IN $\mathrm{BaSnO}_{3}$ : SELF-TRAPPING, TRAPPING BY DOPANTS}

We now describe the oxygen-type hole polaron in BSO, either self-trapped in the lattice (Sec. III B) or trapped next to a dopant (bound polaron, Sec. IIIC). The value of $U$ that minimizes the self-interaction error is, within scheme scheme $\mathrm{o}_{\mathrm{A}}$ (resp. scheme $\mathrm{o}_{\mathrm{C}}$ ), $U=7.5 \mathrm{eV}$ (resp. $4.5 \mathrm{eV}$ ). Within scheme $o_{A}$, we have investigated self-trapping and trapping by dopants using the two values $U=6$ and $8 \mathrm{eV}$, in order to examine how the different properties of the polaron are sensitive to $U$ (the properties at $U=7.5 \mathrm{eV}$ can be estimated by an extrapolation). Within scheme $o_{\mathrm{C}}$, the calculations have been done using $U=4.5 \mathrm{eV}$.

\section{A. Simulating self-trapped small polarons within DFT $+U$ : Methodology}

We wish to place the hole on a precise atom, and also in a precise orbital. It is mandatory to achieve such control because two orbitals are possible per $\mathrm{O}$ atom, that do not drive the same self-trapping distortion, the $2 p$ orbitals being very directional.

For that, we use a numerical functionality implemented in the ABINIT code: within the DFT $+U$ formalism, it is possible to select, and constrain, over a certain number of electronic steps, the configuration of the correlated electrons (those on which $+U$ is applied), by imposing the occupation matrices

\footnotetext{
${ }^{1}$ One assumes for simplicity in these formulas a diagonal density matrix.
} 
TABLE II. Self-trapping energy $(\mathrm{eV})$ of the STHP in $\mathrm{BaSnO}_{3}$, in the different $\mathrm{PBE}+U$ schemes. The value at $U=7.5 \mathrm{eV}$ is a linear extrapolation between $U=6$ and $8 \mathrm{eV}$.

\begin{tabular}{lcccc}
\hline \hline DFT $+U$ scheme & $\mathrm{o}_{\mathrm{A}}$ & & $\mathrm{o}_{\mathrm{A}}$ & $\mathrm{o}_{\mathrm{C}}$ \\
\hline$U(\mathrm{eV})$ & 6 & 7.5 & 8 & 4.5 \\
ST energy & +0.10 & -0.16 & -0.25 & -0.20 \\
\hline \hline
\end{tabular}

of these correlated states [15]. Such procedure has been used up to now to avoid the metastable states in DFT $+U[43,44]$. We use it here to choose the atom and the orbital on which the hole is placed. Practically, we use the following two-step procedure:

(i) A structural optimization is first performed under the constraint of the hole localized on the chosen oxygen atom, in the chosen orbital, using occupation matrices of correlated orbitals which are kept fixed during all this preoptimization. This preliminary step produces a self-trapping distortion around $\mathrm{O}^{-}$favorable to the hole localization on the good atom, and in the good orbital.

(ii) Then, we restart the structural optimization, from this preoptimized geometry, by constraining the correlated electrons only over the first few electronic steps of the first selfconsistent loop, and release completely this constraint afterward. The calculation terminates by relaxing self-consistently the atomic positions and the electrons, with the hole localized on the chosen atom, in the chosen orbital.

We have used such procedure to obtain the isolated STHP in the lattice, and in the vicinity of one of the dopants. The occupation matrices and optimized geometry obtained have then been used to initialize directly the structural optimization of the hole in the case of other dopants.

\section{B. Self-trapped hole in the lattice}

We first focus on the self-trapping energy, i.e., the energy difference between the STHP and the delocalized hole (Table II). Within scheme $\mathrm{o}_{\mathrm{A}}, U=6$ and $8 \mathrm{eV}$ drive, interestingly, two different pictures: the hole polaron is more stable than the delocalized hole using the latter value $(-0.25 \mathrm{eV})$, less stable using the former $(+0.10 \mathrm{eV})$, which implies that, at $U=6 \mathrm{eV}$, the hole might rather diffuse throughout the lattice as a delocalized charge. The linear extrapolation at $U=7.5 \mathrm{eV}$ leads to a self-trapping energy of $-0.16 \mathrm{eV}$, indicating that holes rather have the tendency to form small polarons in barium stannate. Using scheme $\mathrm{o}_{\mathrm{C}}$ with $U=4.5 \mathrm{eV}$, the self-trapping energy is $-0.20 \mathrm{eV}$, which is close to the previous value. The stability of the polaronic state is thus preserved from scheme $o_{A}$ to scheme $o_{C}$.

These values are close to the self-trapping energies of -0.05 and $-0.15 \mathrm{eV}$ computed for oxygen-type hole polarons in $\mathrm{SrTiO}_{3}$ and in $\mathrm{BaCeO}_{3}$ by hybrid functionals [8,9], and to the value of $-0.11 \mathrm{eV}$ obtained by Lindman et al. [4] on $\mathrm{BaZrO}_{3}$ using also $\mathrm{PBE}+U$, with $U$ determined from the piecewise linearity of the energy.

The hole polaron is localized on a $2 p$ orbital perpendicular to the Sn-O-Sn bond and oriented along one axis of the cubic structure. An isosurface of the hole state is shown on Fig. 3(a), in which the hole occupies a $2 p_{x}$ orbital, while the Sn-O-Sn is oriented along $z$. More precisely, this state consists of a $2 p_{x}$ orbital on the $\mathrm{O}^{-}$, slightly hybridized with other $2 p_{z}$ orbitals of the four $\mathrm{O}$ atoms first neighbors in the $(x, z)$ plane. The oxygen atom on which the hole is localized is magnetic, and carries a magnetic moment of $\sim 0.74 \mu_{B}$ (scheme o,$U=8 \mathrm{eV}$ ), while this hybridization leads to a very small magnetic moment on the four oxygen atoms in question, of about $0.04 \mu_{B}$ [46].

There are two possible states for the hole on each oxygen atom [in the case of Fig. 3(a), this would be $2 p_{x}$ and $2 p_{y}$ ]. In the perfect (cubic) lattice, these two states obviously have the same energy, but each one is associated with a specific self-trapping distortion. This is illustrated on Fig. 3(b): as expected, the two $\mathrm{Sn}$ first neighbors are repelled from $\mathrm{O}^{-}$by $0.11 \AA$, while the distance between the four oxygens first neighbors in the $(x, z)$ plane, and $\mathrm{O}^{-}$, is decreased by $\sim 0.10 \AA$. By contrast, the four oxygens first neighbor in the $(y, z)$ plane are almost not moved [Fig. 3(a), inset], showing the strong anisotropy of the self-trapping distortion associated to the hole occupying a very directional $2 p$-type orbital. In $\mathrm{BaZrO}_{3}$, Lindman et al. observe very similar self-trapping distortions [4].

\section{Trapping by dopants}

We now investigate the trapping of the hole polarons by seven trivalent dopants (Ga, Sc, In, Lu, Y, Gd, La) placed in substitution of one Sn (bound polarons). Practically, one $\mathrm{Sn}$ is replaced by the dopant, without additional charge in the supercell. We define the interaction energy between the dopant and the hole $E_{\text {int }}\left(\mathrm{M}_{\mathrm{Sn}}^{\prime}, \mathrm{h}^{\bullet}\right)$, as

$$
\begin{aligned}
E_{\text {int }}\left(\mathrm{M}_{\mathrm{Sn}}^{\prime}, \mathrm{h}^{\bullet}\right)= & E_{\mathrm{tot}}\left(\mathrm{BSO}+\mathrm{M}_{\mathrm{Sn}}, 0\right)+E_{\mathrm{tot}}(\mathrm{BSO}) \\
& -\left\{E_{\mathrm{tot}}\left(\mathrm{BSO}+\mathrm{M}_{\mathrm{Sn}},-1\right)+E_{\mathrm{tot}}(\mathrm{BSO},+1)\right\},
\end{aligned}
$$

where the four terms are, respectively, the total energy of the supercell with one dopant and one hole (with PBE, only the delocalized hole can be considered), that of the perfect supercell, that of the supercell with one dopant (charged -1), and with one hole (charged +1$)$.

We have computed, for each dopant, three different configurations according to the hole-dopant distance: 1st, 2nd, and 3rd neighbor $(\mathrm{N})$, schematically depicted on Fig. 4. The configurations of the hole localized as 1 st and 2nd neighbor close to Y (large dopant) and Ga (small dopant) are shown on Figs. 3(c)-3(f). The presence of the dopant modifies the surrounding distortions with respect to the self-trapped case. In second-neighbor position [Figs. 3(d) and 3(f)], the hole orbital is strongly hybridized with another $2 p$ orbital of the oxygen first neighbor lying in the same plane, and strongly bent in the direction of the dopant, suggesting a strong attractive interaction between the dopant and the hole (a configuration already reported by Lindman el al. in the case of $\mathrm{BaZrO}_{3}$ [4]), and probably a small energy barrier separating the 2 nd $\mathrm{N}$ configuration from the $1 \mathrm{st} \mathrm{N}$ one. Note that in several cases (detailed hereafter), it was not possible to obtain this 2nd $\mathrm{N}$ position, the system evolving to a configuration with the polaron in 1 st $\mathrm{N}$ position.

The dopant-hole interaction energies are gathered in Table III for both DFT $+U$ schemes, for each dopant-hole position, and plotted on Fig. 5 as a function of ionic radius of 
(a) STHP in the lattice

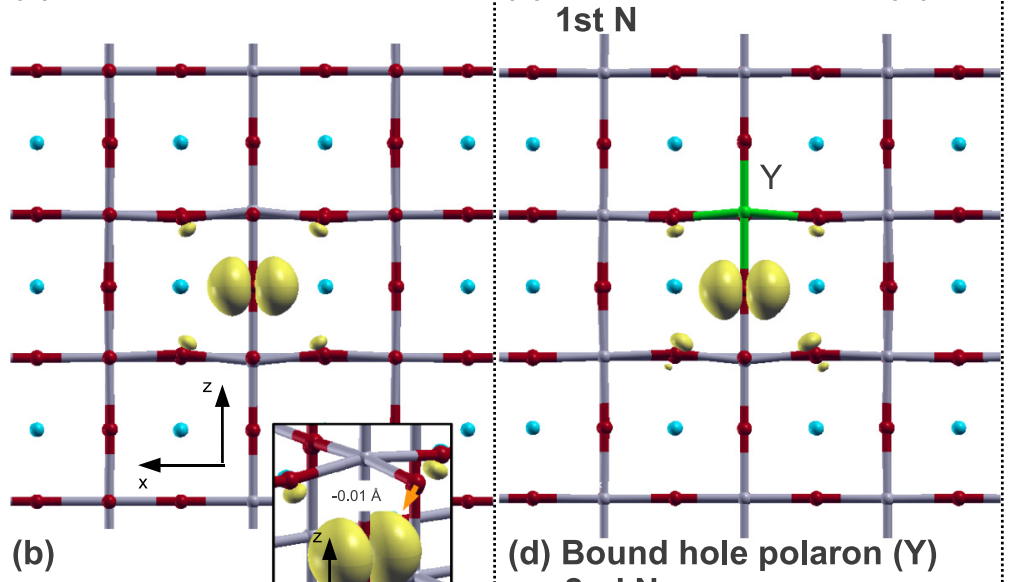

(b)

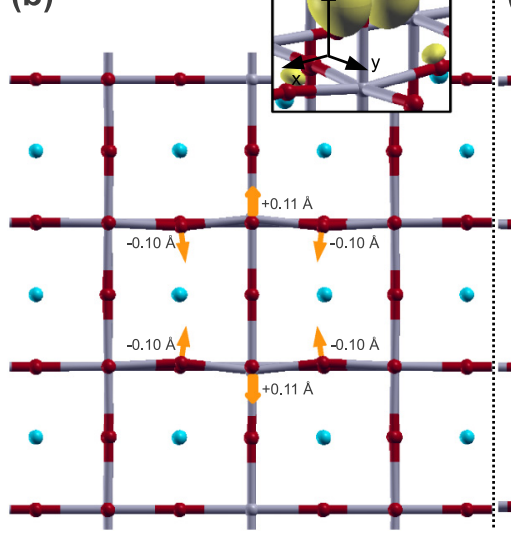

(c) Bound hole polaron (Y) 2nd N (e) Bound hole polaron (Ga) 1st N

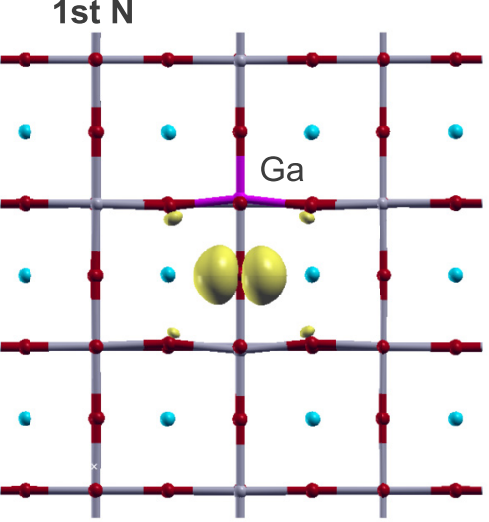

(f) Bound hole polaron (Ga) 2nd N

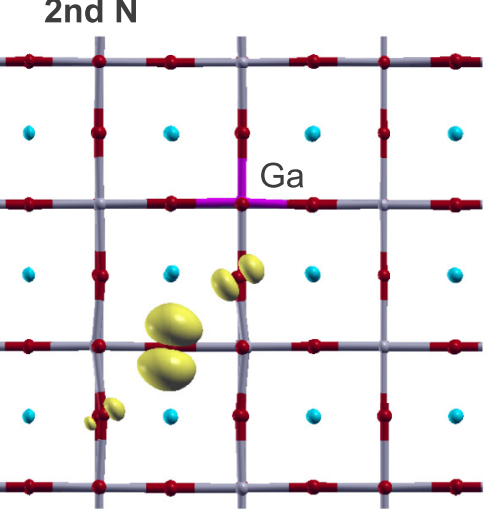

FIG. 3. The oxygen-type hole polaron, in its self-trapped state (a), (b), bound to the yttrium dopant in first-neighbor (c) and second-neighbor (d) position, and to a Ga dopant, in first-neighbor (e) and second-neighbor (f) position. Panels (a), (c), (d), (e), and (f) are isosurfaces of the hole state, while (b) illustrates the lattice distortions associated to the self-trapped hole. Panels (a) and (b) show the self-trapped hole polaron in the lattice (the hole occupies a $2 p_{x}$ orbital), with a self-trapping distortion confined to the $(x, z)$ plane. The insets in (a) and (b) show the quasiabsence of distortion in the $(y, z)$ plane, and thus the anisotropy of the self-trapping distortion. The orange arrows schematically depict the atomic distortions, and the numbers correspond to the variations of the distance between the atom and $\mathrm{O}^{-}$. In second neighbor of the dopant [(d), (f)], the hole has a nonzero probability to be found on the oxygen first neighbor. Figure 3 has been done with the XCRYSDEN software [45].

the dopant $r_{i}$. In 1 st $\mathrm{N}$, the interaction energy globally becomes less negative as $r_{i}$ increases, while in 2 nd $\mathrm{N}$, it becomes more negative, yielding a crossing that takes place close to the ionic radius of $\mathrm{Gd}$. Thus, for the dopants with ionic radii from $\mathrm{Ga}$ to $\mathrm{Y}$, the hole is the most stable as 1 st $\mathrm{N}$ of the dopant (and strongly trapped for the smallest dopants), while in the case of $\mathrm{Gd}$ and $\mathrm{La}$, it is more stable as $2 \mathrm{nd} \mathrm{N}$. It is remarkable that holes obey exactly the same tendency as protons in this oxide [47], at

TABLE III. Dopant-hole interaction energies $E_{\mathrm{int}}\left(\mathrm{M}_{\mathrm{Sn}}^{\prime}, \mathrm{h}^{\bullet}\right)(\mathrm{eV})$ computed in GGA-PBE and GGA-PBE $+U, U=6$ and $8 \mathrm{eV}\left(\mathrm{scheme} \mathrm{o}_{\mathrm{A}}\right)$, and $U=4.5 \mathrm{eV}$ (scheme $\mathrm{o}_{\mathrm{C}}$ ), relative to the self-trapped hole polaron and the ionized dopant isolated in the lattice. The hole polaron has been computed close to the dopant in the three positions shown on Fig. 4, denoted as 1st, 2nd, and 3rd neighbors (N). For PBE, only one value is given that corresponds to the delocalized hole.

\begin{tabular}{|c|c|c|c|c|c|c|c|c|c|c|}
\hline \multirow{4}{*}{$\begin{array}{l}\text { Functional } \\
\text { Scheme } \\
U(\mathrm{eV}) \\
\text { Hole position }\end{array}$} & \multirow[t]{2}{*}{ PBE } & \multicolumn{3}{|c|}{$\mathrm{PBE}+U$} & \multicolumn{3}{|c|}{$\mathrm{PBE}+U$} & \multicolumn{3}{|c|}{$\mathrm{PBE}+U$} \\
\hline & & & $\mathrm{o}_{\mathrm{A}}$ & & & $\mathrm{o}_{\mathrm{A}}$ & & & $\mathrm{o}_{\mathrm{C}}$ & \\
\hline & 0 & & 6 & & & 8 & & & 4.5 & \\
\hline & & $1 \mathrm{st} \mathrm{N}$ & 2nd N & $3 \mathrm{rd} \mathrm{N}$ & 1 st $\mathrm{N}$ & $2 \mathrm{nd} \mathrm{N}$ & $3 \mathrm{rd} \mathrm{N}$ & 1 st $\mathrm{N}$ & 2nd N & $3 \mathrm{rd} \mathrm{N}$ \\
\hline $\mathrm{Ga}$ & -0.16 & -0.51 & - & -0.18 & -0.54 & -0.19 & -0.17 & -0.62 & - & -0.18 \\
\hline $\mathrm{Sc}$ & -0.16 & -0.43 & - & -0.20 & -0.45 & -0.22 & -0.20 & -0.51 & -0.24 & -0.21 \\
\hline In & -0.15 & -0.34 & - & -0.21 & -0.36 & -0.25 & -0.21 & -0.41 & -0.27 & -0.23 \\
\hline $\mathrm{Lu}$ & -0.16 & -0.35 & - & -0.23 & -0.34 & -0.28 & -0.23 & -0.41 & -0.32 & -0.25 \\
\hline $\mathrm{Y}$ & -0.17 & -0.34 & -0.31 & -0.23 & -0.32 & -0.29 & -0.24 & -0.38 & -0.32 & -0.25 \\
\hline $\mathrm{Gd}$ & -0.18 & -0.35 & -0.39 & -0.25 & -0.33 & -0.33 & -0.25 & -0.42 & -0.36 & -0.27 \\
\hline $\mathrm{La}$ & -0.19 & - & -0.48 & -0.27 & -0.27 & -0.44 & -0.28 & -0.34 & -0.51 & -0.30 \\
\hline
\end{tabular}




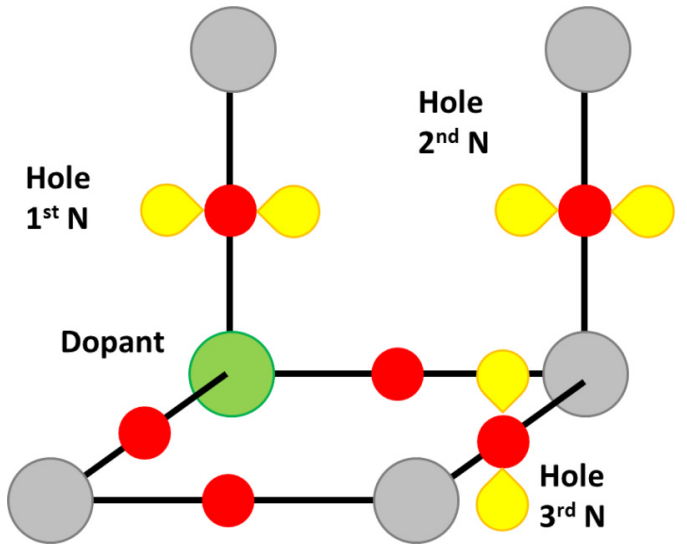

FIG. 4. The three configurations of the hole polaron close to the trivalent dopant. Gray, green, and red circles are, respectively, Sn, dopant, and $\mathrm{O}$ atoms.

the difference that the crossing occurs for protons at a smaller radius, slightly below $0.90 \AA$. Note that using $U=6 \mathrm{eV}$ within scheme $o_{\mathrm{A}}$ does not significantly modify the results, at the exception that, for the dopants with the smaller radii (from $\mathrm{Ga}$ to $\mathrm{Lu}$ ), we did not obtain the 2 nd $\mathrm{N}$ position (the hole
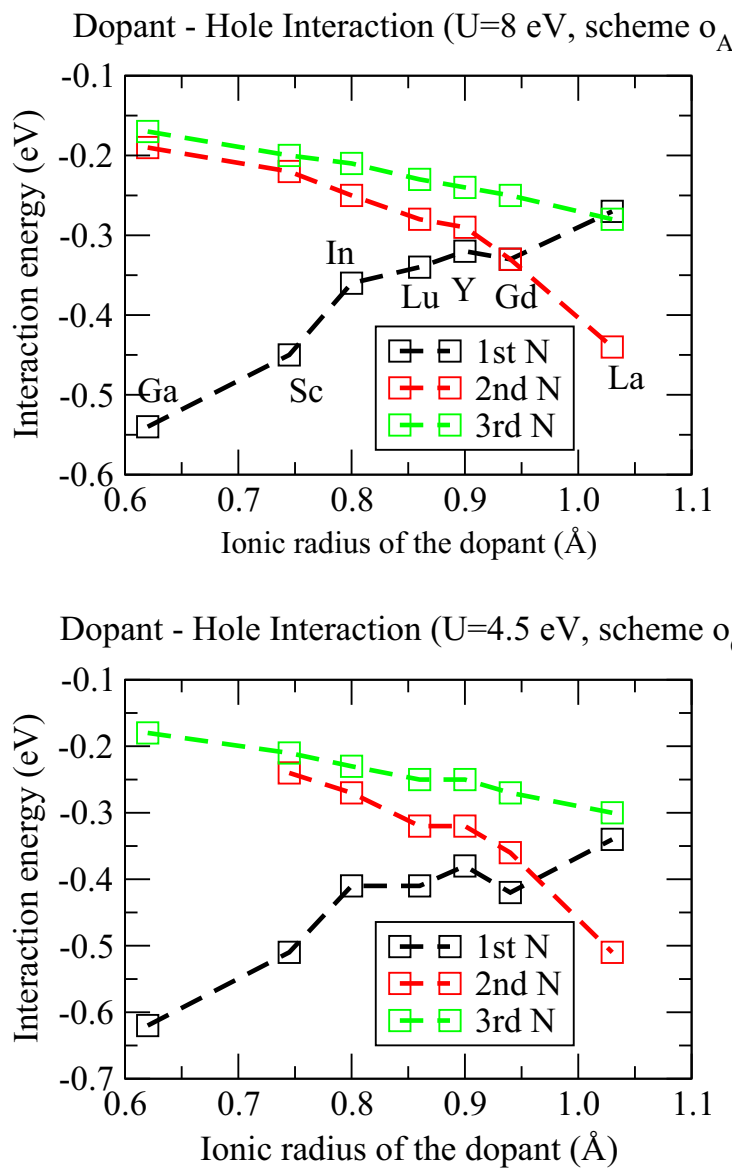

FIG. 5. Dopant-hole interaction energy as a function of ionic radius of the dopant, as obtained with the two DFT $+U$ schemes used in this work. Note that with scheme $o_{C}$, we could not obtain the hole polaron as stable in 2nd neighbor position from the Ga dopant (the configuration evolved to a polaron localized as 1st neighbor). systematically falls in 1st $\mathrm{N}$ position, which is the most stable): the 2 nd $\mathrm{N}$ position was obtained only for $\mathrm{Y}, \mathrm{Gd}$, and $\mathrm{La}$, and the 1 st $\mathrm{N}$ position was not obtained for La. The same problem was encountered for the 2 nd $\mathrm{N}$ position close to $\mathrm{Ga}$ using scheme $o_{C}$. Both $\mathrm{DFT}+U$ schemes yield the same coherent scenario, with trapping energies rather close to each other.

All these configurations, in which the hole is bound to the dopant, are more stable than the delocalized hole, whatever the case. However, at $U=6 \mathrm{eV}$ within scheme $\mathrm{o}_{\mathrm{A}}$, the hole has a mixed behavior: it is localized when trapped in the vicinity of a dopant (and probably up to a rather large distance from it), and itinerant when it is far enough. In other words, it may have an intermediate behavior between localized and more delocalized depending on its proximity to dopants. By contrast, at $U=8 \mathrm{eV}$ within scheme $\mathrm{o}_{\mathrm{A}}$, the hole is localized as a small polaron, whether it is far from a dopant or close to it, in coherence with the picture given by scheme $\mathrm{o}_{\mathrm{C}}$ with $U=4.5 \mathrm{eV}$.

We now comment on the values obtained within scheme $\mathrm{o}_{\mathrm{A}}$. The association energy between the hole and the dopant, that we define as the minimum of the three interaction energies, increases therefore (for $U=8 \mathrm{eV}$ ) from $-0.54 \mathrm{eV}(\mathrm{Ga})$ to $-0.32 /-0.33 \mathrm{eV}(\mathrm{Y} / \mathrm{Gd})$, before redecreasing for La down to $-0.44 \mathrm{eV}$. The dopants commonly employed in BSO (In, $\mathrm{Lu}, \mathrm{Y}, \mathrm{Gd}$ ) exhibit typical dopant-hole association energies $\sim-0.35 /-0.30 \mathrm{eV}$. This trapping energy is rather strong and should be considered when modeling the diffusion of holes throughout the lattice, at least at low temperature (at high temperature, the holes may have escaped from dopants). It is comparable to the association energy between protons and dopants [47]. These values are in line with the trapping energy calculated by Lindman et al. [4] for the hole polaron in $\mathrm{BaZrO}_{3}$ around the $\mathrm{Y}$ dopant $(-0.1$ to $-0.2 \mathrm{eV})$, though a bit more negative: this is probably due to the fact that interaction energies are calculated here with respect to reference systems in which the two defects have no interaction, whereas Lindman et al. use as reference a configuration in which the two defects are as far as possible in the supercell. In real systems where the defect concentration is rather large (typically a few \% or more), our values thus probably overestimate the effective interaction between the defects.

\section{OXYGEN-TYPE HOLE POLARON IN $\mathrm{BaSnO}_{3}$ : MOBILITY}

We now investigate the possible motions of the STHP, isolated in the lattice. Owing to the computational cost of these simulations, we only use here scheme $\mathrm{o}_{\mathrm{A}}$, with $U=8 \mathrm{eV}$, and an atomic data set for $\mathrm{Sn}$ with $4 d$ electrons frozen in the core. We compute the minimum energy paths (MEPs) joining two different optimized polaronic configurations using the simplified string method (SM) [37]. The paths are sampled with 13 images, and the procedure is stopped when the energy difference per image between two consecutive SM steps is lower than $5.0 \times 10^{-5} \mathrm{Ha}$. The SM allows to describe rather sharp and rough energy paths, with possibility of several events occurring along the MEP. Moreover, since the consecutive images are equally distributed along the path, the image index is proportional to the distance along the path, and can therefore be used as a reaction coordinate. We compute the MEPs cor- 

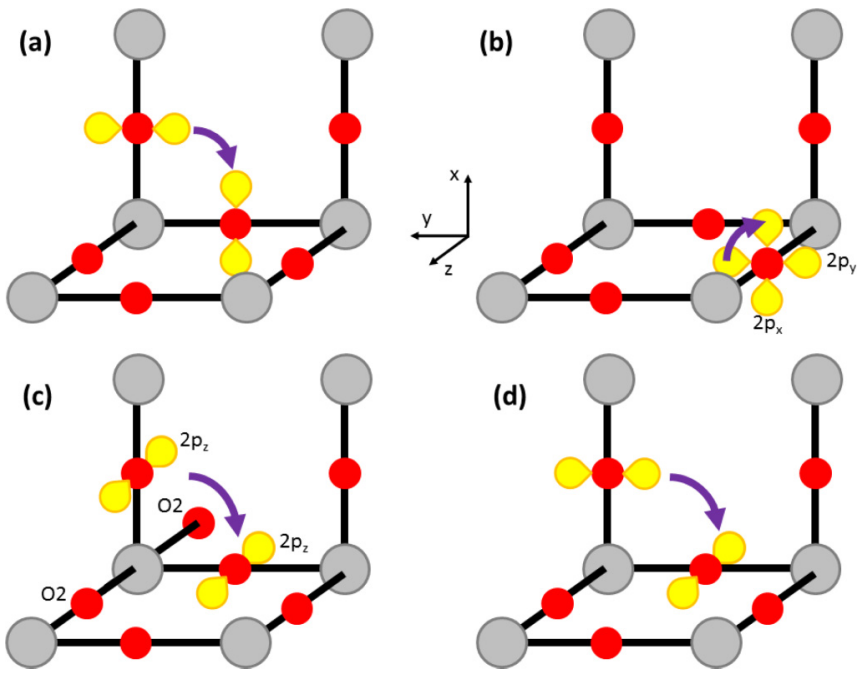

FIG. 6. The four possible motions of the self-trapped hole polaron studied in this work: (a) simple hopping, from the $2 p_{y}$ orbital of an $\mathrm{O}$ atom onto the $2 p_{x}$ orbital of its first-neighbor oxygen; (b) reorientation from the $2 p_{y}$ orbital onto the $2 p_{x}$ orbital of the same $\mathrm{O}$ atom; (c) parallel hopping, from the $2 p_{z}$ orbital of an $\mathrm{O}$ atom onto the $2 p_{z}$ orbital of its first-neighbor oxygen; (d) rotating hopping, from the $2 p_{y}$ orbital of an $\mathrm{O}$ atom onto the $2 p_{z}$ orbital of its first-neighbor oxygen. Gray and red circles are, respectively, Sn and $\mathrm{O}$ atoms.

responding to four different mechanisms, described on Fig. 6. In the standard theory of small polaron hopping [48], the hole polaron may hop, quantum mechanically (tunneling), onto a neighboring site in so-called coincidence configurations (see Appendix B), in which the energy is the same whether the hole is on the initial or on the final site (i.e., the two diabatic ground states are in coincidence). The highest-energy point of the MEP is assumed here as corresponding to the lowest-energy coincidence configuration for the considered mechanism.

The most intuitive motion for the STHP is a hopping onto the $2 p$ orbital of an oxygen first neighbor lying in the same plane [Fig. 6(a), mechanism denoted hereafter as "simple hopping"]. However, such hopping is not sufficient to allow tridimensional long-range diffusion of the hole. Exactly as the diffusion of protons requires the succession of hopping and reorientations, the hole may be able to rotate around its oxygen [4], i.e., change of $2 p$ orbital without hopping onto another atom [Fig. 6(b)]. The two last mechanisms, in which the hole is (i) hopping by staying in an orbital perpendicular to the path ("parallel hopping"), and (ii) hopping and rotating at the same time ("rotating hopping"), have also been investigated [Figs. 6(c) and 6(d)].

\section{A. Simple hopping}

The simple hopping (Fig. 7) exhibits a small barrier of $0.15 \mathrm{eV}$. In the starting geometry, which corresponds to an optimized configuration of the STHP, the magnetic moment on the starting $\mathrm{O}, m_{i}$ is $\sim 0.72 \mu_{B}$ [46], but is not zero on the final O, consistently with the picture provided in Fig. 3(a), in which the STHP has a nonzero density of probability on the four oxygen first neighbors in the plane containing the empty $2 p$ orbital. Along the MEP, $m_{i}$ progressively decreases,
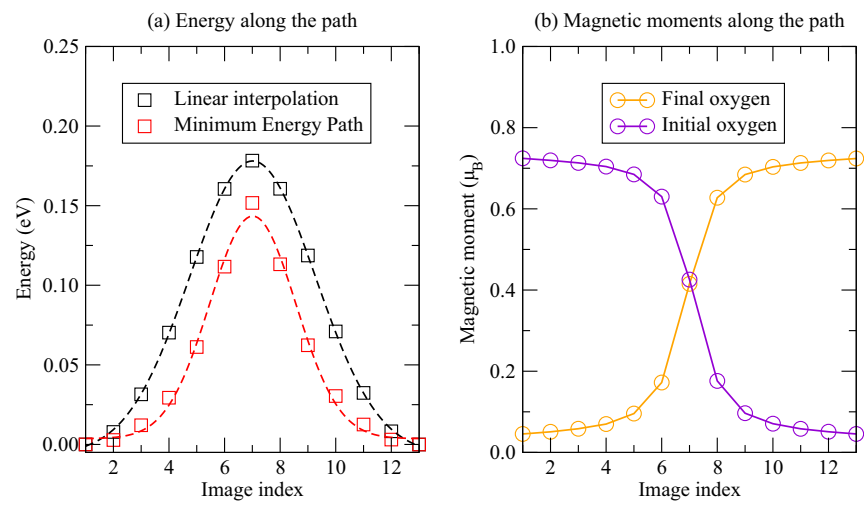

FIG. 7. Simple hopping of the self-trapped hole polaron. (a) Energy along the path (black: linear interpolation, red: minimum energy path from string method calculation). (b) Magnetic moments $\left(\mu_{B}\right)$ of the initial $\left(m_{i}\right)$ and final $\left(m_{f}\right)$ oxygen atoms along the minimum energy path. Dashed lines: Gaussian fit.

while $m_{f}$, the magnetic moment on the final $\mathrm{O}$, progressively increases up to $\sim 0.72 \mu_{B}$ in the final optimized geometry. In the coincidence configuration for hopping, reached at the middle of the MEP, $m_{i} \approx m_{f} \sim 0.4 \mu_{B}$, i.e., the hole is equally shared between the two $O$ atoms. Such behavior, with a very smooth evolution of the hole state, and progressive mixing between the initial and final states along the path, is consistent with an adiabatic hopping (i.e., the charge is progressively transferred with the self-trapping distortion). However, we cannot be sure that this hopping is adiabatic in the present situation because we have no information about the electronic coupling in the coincidence configuration.

The energy barrier obtained along an approximation of the MEP defined by a simple linear interpolation between the initial and final optimized configurations is slightly larger than that of the MEP, $\sim 0.18 \mathrm{eV}$. The energy barrier for hopping, $0.15 \mathrm{eV}$, is in line with values computed for oxygen-type holes in other charge-transfer insulating oxides, e.g., $0.05-0.1 \mathrm{eV}$ in $\mathrm{BaZrO}_{3}$ [4] or $0.16-0.25 \mathrm{eV}$ in $\mathrm{TiO}_{2}$ [16] (case of adiabatic transfers).

\section{B. Reorientation}

The reorientation of the STHP, i.e., its evolution from a $2 p_{y}$ to a $2 p_{x}$ orbital of the same atom, can be treated similarly as hopping because each of the two possible states of the STHP is associated with a specific self-trapping distortion. Here, the magnetic moment cannot be used to follow the evolution of the hole since the hole stays on the same atom. Instead, we use the two diagonal components $\mathrm{O}_{x x}$ and $\mathrm{O}_{y y}$ of the occupation matrix of the $2 p$ states of the $\mathrm{O}$ atom (Fig. 8).

Despite a low-energy barrier, $\sim 0.03 \mathrm{eV}$, the results suggest a strongly nonadiabatic behavior because $\mathrm{O}_{x x}$ and $\mathrm{O}_{y y}$ exhibit a clear discontinuity at the middle of the MEP, where the hole is transferred abruptly from an orbital to the other, whereas the polaronic distortion is, in contrast, progressively transferred.

As a consequence, the point in the middle of the MEP (number 7 on Fig. 8) falls in one of the two electronic states, in contrast, e.g., to what happens for the mechanism of simple hopping, and the SM algorithm produces an asymmetry in the distribution of the images on the final MEP. 

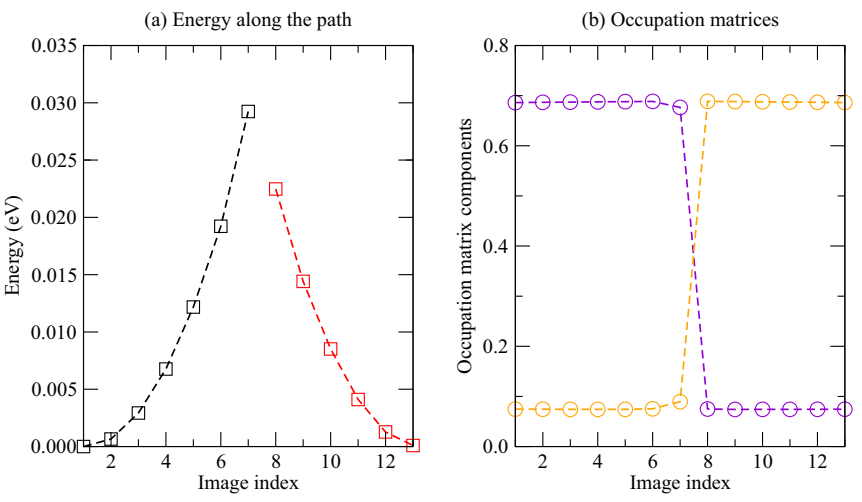

FIG. 8. Reorientation of the hole polaron on a $\mathrm{O}$ atom, from a $2 p_{y}$ to a $2 p_{x}$ orbital. (a) Energy along the minimum energy path (black: hole on $2 p_{y}$, red: hole on $2 p_{x}$ ). (b) Occupation matrix components of the $p$ orbitals on the $\mathrm{O}$ atom along the minimum energy path. Purple: $y y$ component, orange: $x x$ component.

It is, however, possible to restore the symmetry expected at the coincidence configuration, which is done in a separate calculation. Practically, the configuration of image number 7 on the MEP, which is very close to the crossing point, is symmetrized according to the expected space group $(\mathrm{P} 4 / \mathrm{mmm})$. Then, we recompute in this symmetrized configuration the two electronic states, i.e., the hole polaron either in $2 p_{x}$ or in $2 p_{y}$. The two electronic configurations are found to have the same energy with a difference lower than $10 \mu \mathrm{eV}$. Moreover, for the electronic configuration corresponding, for instance, to the hole polaron in the $2 p_{x}$ orbital, the two Kohn-Sham states corresponding to the $2 p_{x}$ and $2 p_{y}$ orbitals have different energies because the electronic density has a broken symmetry, lower than that of the atomic structure. These results suggest that the coincidence point for reorientation of the hole is a Born-Oppenheimer crossing point.

\section{Parallel hopping}

In the parallel hopping mechanism (Fig. 9), the MEP exhibits a barrier of $0.35 \mathrm{eV}$, significantly larger than the two previous ones. In the coincidence configuration, the hole
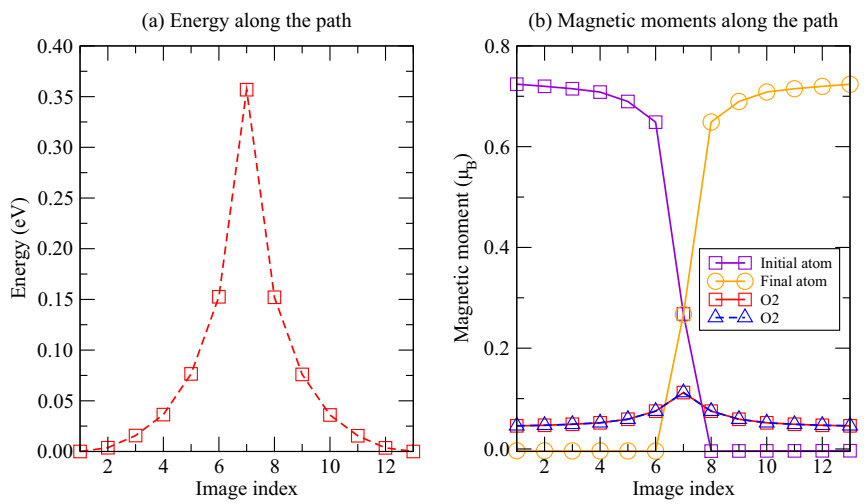

FIG. 9. Parallel hopping of the hole polaron, from a $2 p_{z}$ to a $2 p_{z}$ orbital. (a) Energy along the minimum energy path; (b) magnetic moments $\left(\mu_{B}\right)$ of the initial $\left(m_{i}\right)$ and final $\left(m_{f}\right)$ oxygen atoms along the minimum energy path.
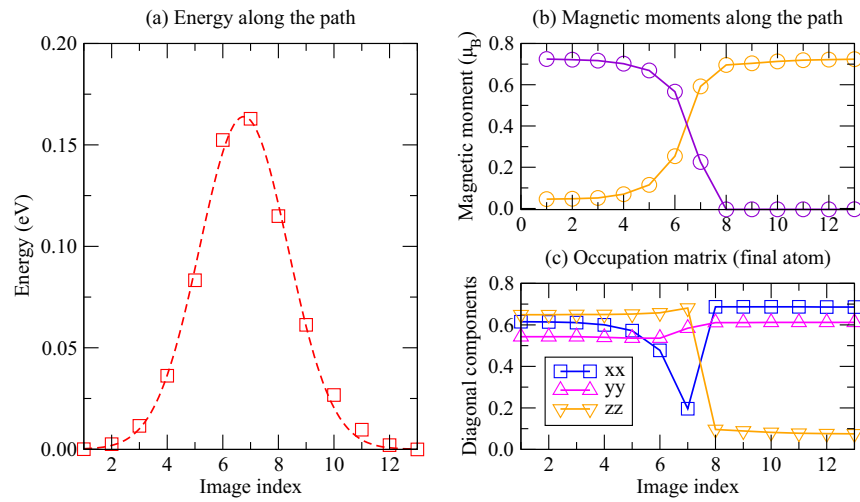

FIG. 10. Rotating hopping of the self-trapped hole polaron. (a) Energy along the MEP; (b) magnetic moments $\left(\mu_{B}\right)$ of the initial $\left(m_{i}\right)$ and final $\left(m_{f}\right)$ oxygen atoms along the minimum energy path; (c) diagonal components of the $p$ orbital occupation matrix on the final atom [see Fig. 6(d)].

is equally shared between the two $\mathrm{O}$ atoms (which have a magnetic moment of $\sim 0.27 \mu_{B}$ ), but a significant part of it is localized on the two $\mathrm{O}$ atoms labeled as $\mathrm{O} 2$ on Fig. 6(c) $\left(\sim 0.10 \mu_{B}\right.$ per $\left.\mathrm{O} 2\right)$. In other words, part of the hole is transferring via the two $\mathrm{O} 2$ oxygen atoms.

On the one hand, the hole charge is continuously transferred from the initial atom onto the final one and moves with the atomic distortion, but on the other hand, the two profiles of Fig. 9 are much less smooth than the ones of "simple hopping," suggesting for this parallel hopping a behavior intermediate between simple hopping and reorientation.

\section{D. "Rotating hopping"}

The last mechanism (Fig. 10) corresponds to a hopping of the hole polaron, from one $\mathrm{O}$ atom onto another (1st $\mathrm{N})$, but the final orbital of the hole does not lie in the same plane as initially [Fig. 6(d)]. Although the hopping distance is the same as for simple hopping, the initial and final states obviously have a smaller overlap, suggesting lower electronic coupling between initial and final states. However, the optimized MEP provides a barrier of $0.16 \mathrm{eV}$, close to that of the simple hopping mechanism $(0.15 \mathrm{eV})$. In fact, this mechanism is simply the succession of a simple hopping and a reorientation, as it can be seen on Fig. 10: the hole first progressively transfers from the $2 p_{y}$ of the initial atom onto the $2 p_{x}$ of the final one, and then abruptly rotates onto the the $2 p_{z}$, with a sharp discontinuity in the occupation matrices.

\section{HOLE DIFFUSION IN THE LATTICE}

The results may be used to draw a picture of hole diffusion in BSO. In their most stable state, holes are trapped around dopants. This trapping probably slows down their motions, at least at low dopant concentration and low temperature, as for protons, for which the trapping effect by negative dopants is well documented $[47,49,50]$. However, our calculations drive two different models for hole diffusion far from the dopants, according to the DFT $+U$ scheme and value of $U$ :

(i) $U=6 \mathrm{eV}\left(\right.$ scheme $\left.\mathrm{o}_{\mathrm{A}}\right)$ : the delocalized hole is more stable than the STHP in the lattice, and the hole is localized 
only when trapped around dopants. When the hole has escaped from the attraction of the dopant, it tends to delocalize, and moves throughout the lattice as a free particle. The system would be here rather similar to an extrinsic $p$-type semiconductor, at the exception that the trapping energy of the holes may be as large as $\sim 0.3-0.5 \mathrm{eV}$ (depending on the dopant), and their concentration in the lattice determined by the free enthalpy of the oxidation reaction.

(ii) $U=8 \mathrm{eV}$ (scheme $\mathrm{o}_{\mathrm{A}}$ ): the STHP is always more stable than the delocalized hole, whether trapped or selftrapped, suggesting that diffusion occurs by a succession of hoppings, with the hole being always under the form of a small polaron. Scheme o $o_{C}$ with $U=4.5 \mathrm{eV}$ is consistent with this picture.

The value of $U$ predicted by the piecewise linearity of the energy provides a picture corresponding to this second model, with an itinerant level $0.16-0.20 \mathrm{eV}$ higher than the STHP.

The simple hopping mechanism allows diffusion of the hole inside a $\{100\}$ plane only, and is thus not enough to explain its tridimensional long-range diffusion. The hole, to be able to migrate on long distances, should be able to change plane, either by rotating (reorientation/rotating hopping) or by parallel hopping. However, it remains undetermined, from our calculations, whether the reorientation process is more likely to occur than the parallel hopping processes, owing to its possible nonadiabatic character (despite a very low activation energy, the transition rate for reorientation could be considerably lowered by a possible very low prefactor). The activation energy for hole mobility far from dopants (i.e., independently of the trapping effect) is thus at least the value obtained in the case of simple hopping, i.e., $0.15 \mathrm{eV}$, and at most that obtained with parallel hopping, i.e., $0.35 \mathrm{eV}$. At high temperature, it is likely that only holes which have escaped from dopants contribute to the conductivity, providing a rather small activation energy for hole migration between 0.15 and $0.35 \mathrm{eV}$.

Alternatively, the hole mobility could be dominated by the few holes thermally excited in the itinerant level [12].

\section{CONCLUSION}

In this work, we have performed density functional theory calculations, in the GGA $+U$ framework, with a Hubbard correction applied on the $p$ states of oxygen, to model oxygen-type holes in acceptor-doped barium stannate. Using a $U$ determined from the piecewise linearity of the energy, holes are found localized as small polarons in $\mathrm{BaSnO}_{3}$, with a self-trapping energy of $\sim-0.2 \mathrm{eV}$. Close to negative dopants, the holes are localized, and trapped, with trapping energies typically $\sim-0.3 /-0.4 \mathrm{eV}$. The hole-dopant association energy behaves like the proton-dopant association energy [47]: the hole polaron is trapped at oxygen 1st neighbors for small dopants, while it is trapped at an oxygen 2 nd neighbor for large dopants, the crossing occurring for a dopant ionic radius close to that of $\mathrm{Gd}$.

The hole mobility has been modeled on the basis of four possible motions (three hoppings and a rotation, or reorientation), that strongly differ from each other: in simple hopping, the hole charge is continuously transferred along the minimum energy path, together with the atomic distortions, whereas this is not the case in the reorientation process. The rotating hop- ping consists of a simple hopping followed by a reorientation and, thus, exhibits the same behavior as reorientation. Finally, the parallel hopping probably has a behavior intermediate between simple hopping and reorientation. It follows that hole migration is a complex process, with an activation energy $E_{h}$ between 0.15 and $0.35 \mathrm{eV}$, depending on the possibility of occurrence of reorientations, and on the trapping by dopants. At high temperature, where barium stannate is likely to be oxidized [Eq. (1)], the holes contributing to the conductivity have probably escaped from dopants. Therefore, a rather small activation energy for hole migration is expected. This is in line with the conclusions of Ref. [4] on $\mathrm{BaZrO}_{3}$.

A slightly modified DFT $+U$ scheme is proposed, of particular importance for the $p$ orbital of oxygen. This scheme leads to use more physical values of $U$, lower than with the standard scheme, and describes well the properties of the hole polaron.

Note added in proof. Recently, we became aware of a related work of Wang and coworkers [55], which emphasizes also the impact of the projection radius in $\mathrm{DFT}+U$ for polaron properties.

\section{ACKNOWLEDGMENTS}

We thank F. Jollet for useful discussions about PAW atomic data, as well as Y. Bronstein, Ph. Depondt, and F. Finocchi for fruitful discussions about hole hoppings and rotations. $\mathrm{X}$. Gonze is acknowledged for a stimulating discussion about polarons. The calculations have been performed on the TERA100 supercomputer of CEA/DAM.

\section{APPENDIX A: SEMICORE ELECTRONS, PAW RADII OF ATOMIC DATA SETS}

Details about semicore electrons and PAW radii are given in Table IV. The atomic data sets for Ga, Sc, Lu, Y, Gd, and La are taken from the JTH02 table [51]. Gd is treated as spin polarized.

\section{APPENDIX B: HOLE POLARONS IN CHARGE-TRANSFER INSULATING OXIDES}

We recall here a few basic concepts about small polarons in crystals [48], with emphasis on oxygen-type hole polarons in perovskites. Small polarons are obtained when an excess charge (electron or hole), typically consecutive to the forma-

TABLE IV. Atomic data sets used in this work: valence electrons and PAW radius.

\begin{tabular}{lcc}
\hline \hline Element & Electrons & PAW radius (a.u.) \\
\hline $\mathrm{Ba}$ & $5 s^{2} 5 p^{6} 6 s^{2}$ & 2.4 \\
$\mathrm{Sn}$ & $4 d^{10} 5 s^{2} 5 p^{2}$ & 2.3 \\
$\mathrm{O}$ & $2 s^{2} 2 p^{4}$ & $1.4 / 1.6$ \\
$\mathrm{Ga}$ & $3 d^{10} 4 s^{2} 4 p^{1}$ & 2.1 \\
$\mathrm{Sc}$ & $3 s^{2} 3 p^{6} 4 s^{2} 3 d^{1}$ & 2.4 \\
$\mathrm{In}$ & $4 d^{10} 5 s^{2} 5 p^{1}$ & 2.3 \\
$\mathrm{Lu}$ & $5 s^{2} 5 p^{6} 6 s^{2} 5 d^{1} 4 f^{14}$ & 2.5 \\
$\mathrm{Y}$ & $4 s^{2} 4 p^{6} 5 s^{2} 4 d^{1}$ & 2.2 \\
$\mathrm{Gd}$ & $5 s^{2} 5 p^{6} 6 s^{2} 5 d^{1} 4 f^{7}$ & 2.5 \\
$\mathrm{La}$ & $5 s^{2} 5 p^{6} 6 s^{2} 5 d^{1} 4 f^{0}$ & 2.5 \\
\hline \hline
\end{tabular}


Undistorted lattice

(a)

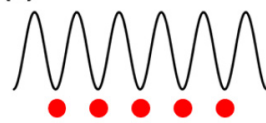

(c)

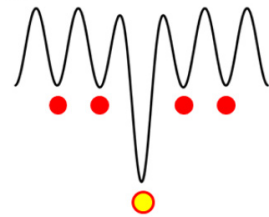

(b)

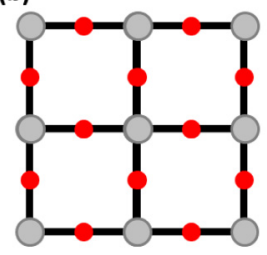

(d)

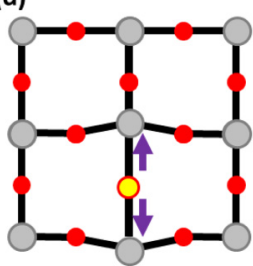

(e)

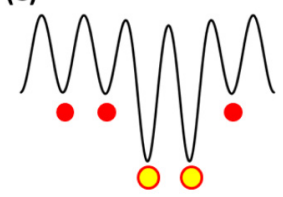

(f)

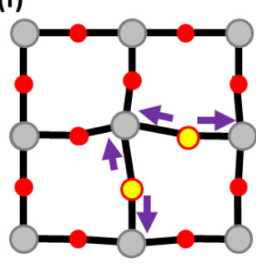

FIG. 11. Schematic representation of a small polaron in a crystal. Upper panels: schematic view of the potential felt by the excess charge; lower panels: application to the oxygen-type hole polaron in a perovskite oxide (only one plane of the $\mathrm{SnO}_{2}$ sublattice is shown). (a), (b) Undistorted lattice, all the $\mathrm{O}$ sites (red circles) are equivalent, the hole is delocalized. (c), (d) Self-trapped configuration: the hole polaron is localized on the yellow $\mathrm{O}$ atom. It repels the two nearestneighbor Sn atoms (purple arrows), which contribute to stabilize its site. (e), (f) Coincidence configuration for hopping: the hole has the same energy whether it is placed on a yellow $\mathrm{O}$ or on the other.

tion of a point defect (donor or acceptor), in a polarizable insulating lattice localize on one single atom instead of being delocalized throughout the crystal. Such localization is concomitant with a polarization distortion of the surrounding matrix (called "self-trapping" distortion) that contributes to create on the site in question a deep electrostatic potential favorable to the localization of the charge [Figs. 11(c) and 11(d)]. This electrostatic energy counterbalances the cost associated with the quantum confinement of the charge. The localized charge and its self-trapping distortion cannot be dissociated from each other. Both of them form the entity commonly called as "small polaron."

In the case of an oxygen-type STHP in a perovskite oxide, the self-trapping distortion mainly corresponds to the two B ions first neighbor being repelled [Fig. 11(d)] and four oxygens first neighbor being slightly attracted, in the plane defined by the B-O-B bond and the $2 p$ orbital of the hole.

The migration of small polarons is a thermally activated phenomenon. However, such thermal activation does not result from the thermal overbarrier motion of the electronic charge itself. The motion of the charge is indeed, intrinsically, a tunneling process. But, such tunneling is not likely to take place in the stable, self-trapped, configuration because the charge is strongly trapped in the potential well caused by the surrounding self-trapping distortion, making the neighboring sites of high energy with respect to the self-trapped site [Figs. 11(c) and 11(d)]. For such tunneling to occur, it is necessary that the crystal around be distorted until one neighboring site has the same energy as the polaron site, producing degenerate (diabatic) energy levels, a state called "coincidence configuration" [48] [Figs. 11(e) and 11(f)]. Such distortion can be produced by the thermal agitation of the surrounding atoms, with a probability $\propto e^{-E_{c} / k_{B} T}$, where
$E_{c}$ is the coincidence energy, i.e., the energy of the most stable coincidence configuration with respect to the stable self-trapped one. The coincidence energy $E_{c}$ plays the role of an activation energy for the hopping process of the polaron.

The hopping rate for the small polaron hopping is thus $\propto e^{-E_{c} / k_{B} T}$, but the energy in this Arrhenius term, to be paid by thermal agitation, is related to the motions of the surrounding atoms, not to the motion of the electronic charge itself. According to the typical tunneling probability in the coincidence configuration, compared to the time scale of the coincidence, the transfer can be adiabatic (i.e., the electronic charge has the time to tunnel), nonadiabatic (i.e., the electronic charge has not the time to tunnel, so that many occurrences of the coincidence are necessary before a jump occurs), or lie in-between. In an adiabatic hopping, the charge is progressively transferred from the initial to the final site, together with the self-trapping distortion [4].

\section{APPENDIX C: DFT $+U$ AND O- $p$ ORBITALS}

This appendix intends to deepen the explanation concerning the calculation of the density matrix in PAW. We first remind the basic equation of DFT $+U$ in PAW, and present expression for the renormalization of the density matrix as detailed in Sec. II B.

As seen above, such renormalization enables to obtain reasonable and physical values of $U$ while preserving the physical properties of the hole polaron (self-trapping, trapping energies). An important aspect of DFT $+U$ calculations is the definition of correlated orbitals. Indeed, DFT $+U$, as well as the DFT+DMFT method, is dependent on their definition: it was recently highlighted $[52,53]$ that using different definitions and different implementations lead to important differences in transition-metal oxides. Importantly, the number of correlated electrons is very sensitive to this choice, and thus the total energy, and thus the effect of DFT $+U$ to restore the piecewise linearity of the total energy as a function of the number of electrons [15] (see discussion in Sec. II C).

We thus first focus on the definition of the correlated orbitals: most DFT $+U$ calculations on hole polaron used the same implementation [39,54], in which the correlated orbitals are not directly defined and occupation matrices are computed thanks to Eq. (20) of Ref. [53]:

$$
n_{m, m^{\prime}}^{l, \sigma}=\sum_{n n^{\prime}} \rho_{(m, n),\left(m^{\prime}, n^{\prime}\right)}^{l, \sigma}\left\langle\phi_{n}^{t} \mid \phi_{n^{\prime}}^{t}\right\rangle, \quad\left[\text { scheme } \mathrm{o}_{\mathrm{A}}\right]
$$

where $m, m^{\prime}$ are the quantum numbers related to the projection of the angular momentum and $n, n^{\prime}$ are index for PAW projectors. $\rho_{(m, n),\left(m^{\prime}, n^{\prime}\right)}^{l, \sigma}$ is defined in, e.g., Ref. [40]. Importantly, in this definition, scalar products are carried out only inside PAW atomic sphere as discussed in Ref. [40]. This is the scheme called "PAW" in Ref. [53]. A nearly identical formulation in terms of occupation matrix can be obtained by defining correlated orbitals as truncated renormalized atomic orbitals. Let us call $\left|\phi_{0}^{t}\right\rangle$ a truncated $(t)$ unnormalized atomic orbital, as used in the PAW scheme. We define a renormalized orbital as $\left|\bar{\phi}_{0}^{t}\right\rangle=\frac{\left|\phi_{0}^{t}\right\rangle}{\sqrt{\left\langle\phi_{0}^{t} \mid \phi_{0}^{t}\right\rangle}}$. The occupation matrix thus reads as

$$
\left.n_{m, m^{\prime}}^{l, \sigma}=\sum_{n n^{\prime}} \rho_{(m, n),\left(m^{\prime}, n^{\prime}\right)}^{l, \sigma}\left\langle\phi_{n}^{t} \mid \bar{\phi}_{0}^{t}\right\rangle\left\langle\bar{\phi}_{0}^{t} \mid \phi_{n^{\prime}}^{t}\right\rangle . \quad \text { [scheme } \mathrm{o}_{\mathrm{B}}\right]
$$


TABLE V. For the three occupation matrices presented in Eqs. (C1), (C2), and (C4), the table gives the number of $p$ electrons $n_{p}$ in $\mathrm{BaSnO}_{3}$, computed with $U=4.5 \mathrm{eV}$ and $J=0 \mathrm{eV}$, the mathematical maximum possible number of electrons $n_{\max }$ (corresponding to an infinite Fermi level), and the Kohn-Sham gap. The difference between $n_{p}$ and $n_{\max }$ comes from the hybridization between oxygen and metal atoms. In a purely ionic limit, we should thus have $n_{p}=n_{\max }$. Occupation matrices $\mathrm{o}_{\mathrm{A}}$ and $\mathrm{o}_{\mathrm{B}}$ give identical results. Only the occupation matrix $o_{C}$ is able to recover the physical exact maximum number of $p$ electrons, and thus has a correct number of electrons $n_{p}$.

\begin{tabular}{lccc}
\hline \hline Occupation matrix & $n_{p}$ & $n_{\max }$ & Gap (eV) \\
\hline $\mathrm{o}_{\mathrm{A}}$ & 3.64 & 4.44 & 1.36 \\
$\mathrm{o}_{\mathrm{B}}$ & 3.63 & 4.44 & 1.36 \\
$\mathrm{o}_{\mathrm{C}}$ & 4.99 & 6.00 & 2.16 \\
\hline \hline
\end{tabular}

Let us assume that one has only one projector, and that this projector corresponds to the atomic O- $p$ eigenstates. Thus, $\phi_{n}^{t}=\phi_{n^{\prime}}^{t}=\phi_{0}^{t}$ and the two formulations are equivalent. The assumption of only one projector is not drastic in localized systems because the energy dispersion is weak. So, we established that occupation matrices obtained from Eqs. (C1) and (C2) are equivalent. This is confirmed by direct calculation of the number of electrons and gap reproduced on Table V.

Going back to the original definition of the occupation matrix, Eq. (C2) is thus equivalent to

$$
n_{m, m^{\prime}}^{l, \sigma}=\sum_{\mathbf{k}, v} f_{v}^{\mathbf{k}, \sigma}\left\langle\Psi_{v}^{\mathbf{k}, \sigma} \mid Y_{l m^{\prime}} \bar{\phi}_{0}^{t}\right\rangle\left\langle Y_{l m} \bar{\phi}_{0}^{t} \mid \Psi_{v}^{\mathbf{k}, \sigma}\right\rangle,
$$

where the scalar product is still done only inside atomic sphere.

As discussed in Sec. II B, in order to recover the physical number of electrons in the correlated orbitals, one needs to renormalize the density matrix, using

$$
\begin{aligned}
n_{m, m^{\prime}}^{l, \sigma}= & \sum_{\mathbf{k}, v} f_{v}^{\mathbf{k}, \sigma}\left\langle\Psi_{v}^{\mathbf{k}, \sigma} \frac{\left|Y_{l m^{\prime}} \bar{\phi}_{0}^{t}\right\rangle\left\langle Y_{l m} \bar{\phi}_{0}^{t}\right|}{\left\langle\phi_{0}^{t} \mid \phi_{0}^{t}\right\rangle} \Psi_{v}^{\mathbf{k}, \sigma}\right\rangle \\
= & \sum_{\mathbf{k}, v} f_{v}^{\mathbf{k}, \sigma}\left\langle\Psi_{v}^{\mathbf{k}, \sigma} \frac{\left|Y_{l m^{\prime}} \phi_{0}^{t}\right\rangle\left\langle Y_{l m} \phi_{0}^{t}\right|}{\left(\left\langle\phi_{0}^{t} \mid \phi_{0}^{t}\right\rangle\right)^{2}} \Psi_{v}^{\mathbf{k}, \sigma}\right\rangle . \\
& {[\text { scheme o }] }
\end{aligned}
$$

With this definition, one recovers the same number of electrons as if we would use an untruncated atomic orbital in place of a renormalized truncated orbital in Eq. (C2). Indeed, one can easily show that, for, e.g., an atom or a simple heteronuclear diatomic molecule in the linear combination of atomic orbital assumption, the simple expression of Eq. (C4) gives the correct number of electrons (see also Table V). We thus emphasize that this renormalization keeps the essential and important physics of hybridization. On contrary, schemes $\mathrm{o}_{\mathrm{A}}$ [Eq. (C1)] and $\mathrm{o}_{\mathrm{B}}$ [Eq. (C2)] do not even reproduce the correct number of electrons in atomic oxygen. The formulation of Eq. (C4) is thus equivalent to the "ATOMIC" scheme in Ref. [53]. An important point is indeed that this formulation can recover the maximum number of electrons in oxygen orbitals, in contrary to other schemes, as can be seen in Table V. So, the scheme $\mathrm{o}_{\mathrm{C}}$ [Eq. (C4)] is much more physical than schemes o $\mathrm{o}_{\mathrm{A}}$ [Eq. (C1)]
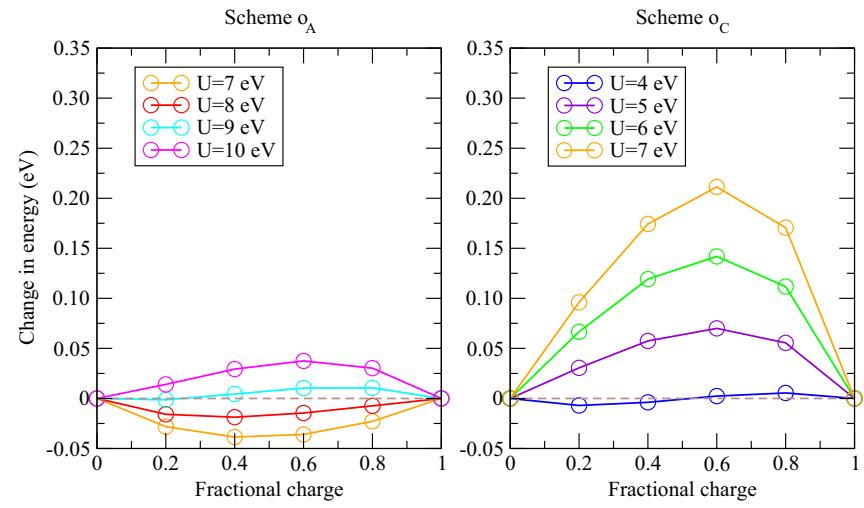

FIG. 12. Energy change with respect to piecewise linearity, as a function of fractional charge (number of electrons) in the polaron, using an oxygen atomic data set with a radius of 1.4 a.u. The linearity is subtracted so that the concavity or convexity appears clearly. Left panel: standard DFT $+U$ scheme $\left(\mathrm{o}_{A}\right)$, with $U=7,8,9$, and $10 \mathrm{eV}$; right panel: scheme $\mathrm{o}_{\mathrm{C}}(U=4,5,6$, and $7 \mathrm{eV})$. The atomic geometry (positions, lattice constant) is the same as in Fig. 2.

and $\mathrm{o}_{\mathrm{B}}$ [Eq. (C2)], as used in the literature. The difference is noticeable for oxygen- $p$ orbitals, but weak for $d$ and $f$ orbitals for which atomic orbitals are mainly inside the atomic sphere.

\section{APPENDIX D: IMPACT OF PAW ATOMIC RADIUS}

In this appendix, we scrutinize the impact of the oxygen PAW radius on the best value of $U$ and on the self-trapping energy, using both DFT $+U$ schemes. The piecewise linearity curves are recomputed using an oxygen PAW atomic data set with a radius of 1.4 a.u., and displayed on Fig. 12. Using scheme $\mathrm{o}_{\mathrm{A}}$, the shorter radius enhances $U$ by more than $1 \mathrm{eV}$, as expected, providing a $U$ between 8 and $9 \mathrm{eV}$ (closer to $9 \mathrm{eV}$ ). However, scheme $\mathrm{o}_{\mathrm{C}}$ provides a $U$ very close to $4 \mathrm{eV}$, thus closer to the one obtained using the previous oxygen pseudopotential.

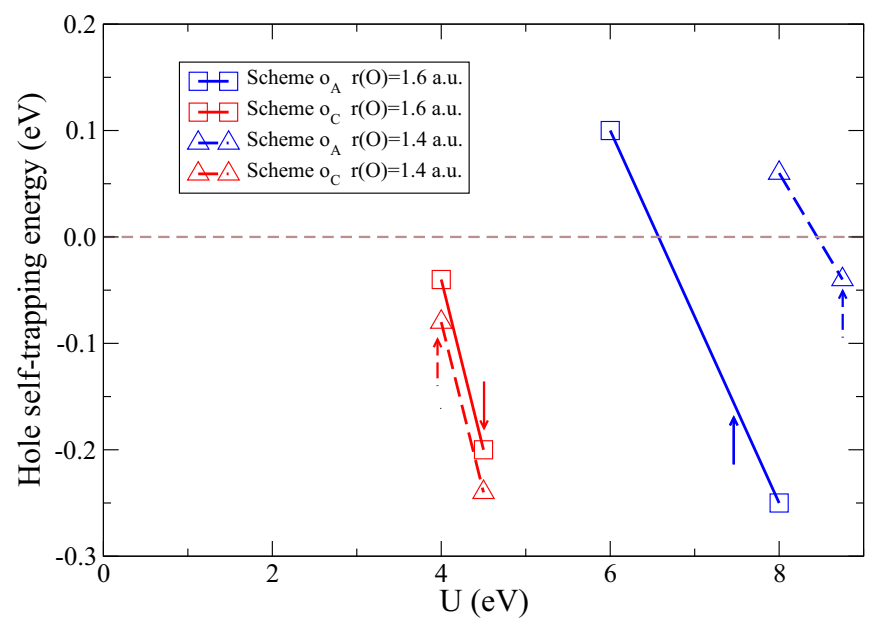

FIG. 13. Hole self-trapping energy $(\mathrm{eV})$ as a function of $U(\mathrm{eV})$, in the two DFT $+U$ schemes, and using two oxygen atomic data sets with two different radii (1.6 and 1.4 a.u.). The arrow indicates, for each scheme, the approximate best value of $U$ as dictated by piecewise linearity of the energy. 
The self-trapping energies have been recomputed with this new oxygen PAW data set using older $U$ and other values corresponding more closely to the piecewise linearity of the energy. They are plotted on Fig. 13 using both DFT $+U$ schemes, as a function of $U$. From this figure, it is manifest that at fixed $U$, the self-trapping energy is strongly modified when the radius is changed in scheme $\mathrm{o}_{\mathrm{A}}$, but much less in scheme $o_{\mathrm{C}}$. However, the slope of the ST energy versus $U$ curve remains large. Thus, owing to the uncertainty we have on the best possible $U$ (a few $0.1 \mathrm{eV}$ ), it is not possible to accurately quantify the dependency of the ST energy itself on the pseudopotential radius.
[1] K. D. Kreuer, Solid State Ionics 125, 285 (1999).

[2] T. He, K.-D. Kreuer, Y. M. Baikov, and J. Maier, Solid State Ionics 95, 301 (1997).

[3] J. B. Varley, A. Janotti, C. Franchini, and C. G. Van de Walle, Phys. Rev. B 85, 081109(R) (2012).

[4] A. Lindman, P. Erhart, and G. Wahnström, Phys. Rev. B 94, 075204 (2016).

[5] K. Kobayashi, S. Yamaguchi, and Y. Iguchi, Solid State Ionics 108, 355 (1998).

[6] R. Glöckner, M. S. Islam, and T. Norby, Solid State Ionics 122, 145 (1999).

[7] S. J. Stokes and M. S. Islam, J. Mater. Chem. 20, 6258 (2010).

[8] A. Janotti, J. B. Varley, M. Choi, and C. G. Van de Walle, Phys. Rev. B 90, 085202 (2014).

[9] M. Swift, A. Janotti, and C. G. Van de Walle, Phys. Rev. B 92, 214114 (2015).

[10] E. Bevillon, G. Dezanneau, and G. Geneste, Phys. Rev. B 83, 174101 (2011).

[11] J. Hermet, F. Bottin, G. Dezanneau, and G. Geneste, Phys. Rev. B 85, 205137 (2012).

[12] A. Lindman, P. Erhart, and G. Wahnström, Phys. Rev. B 91, 245114 (2015).

[13] P. Erhart, A. Klein, D. Aberg, and B. Sadigh, Phys. Rev. B 90, 035204 (2014).

[14] K. Hoang, Phys. Rev. Appl. 3, 024013 (2015).

[15] M. Cococcioni and S. de Gironcoli, Phys. Rev. B 71, 035105 (2005).

[16] N. A. Deskins and M. Dupuis, J. Phys. Chem. C 113, 346 (2009).

[17] B. J. Morgan and G. W. Watson, Phys. Rev. B 80, 233102 (2009).

[18] S. Lany and A. Zunger, Phys. Rev. B 80, 085202 (2009).

[19] Y. Wang, A. Chesnaud, E. Bevillon, J. Yang, and G. Dezanneau, Mater. Sci. Eng. 176, 1178 (2011).

[20] Y. Wang, A. Chesnaud, E. Bevillon, and G. Dezanneau, Solid State Ionics 214, 45 (2012).

[21] Y. Wang, A. Chesnaud, E. Bevillon, J. Xiong, and J. Yang, J. Alloys Compd. 555, 395 (2013).

[22] Y. Wang, T. Su, W. Liu, Y. Dong, Q. Chang, and G. Qiao, Ceram. Int. 41, 481 (2015).

[23] T. Schober, Solid State Ionics 109, 1 (1998).

[24] P. Murugaraj, K. D. Kreuer, T. He, T. Schober, and J. Maier, Solid State Ionics 98, 1 (1997).

[25] Y. Wang, A. Chesnaud, E. Bevillon, J. Yang, and G. Dezanneau, Int. J. Hydrogen Energy 36, 7688 (2011).

[26] L. Buannic, F. Blanc, D. S. Middlemiss, and C. P. Grey, J. Am. Chem. Soc. 134, 14483 (2012).

[27] L. Li and J. C. Nino, Int. J. Hydrogen Energy 38, 1598 (2013).

[28] F. G. Kinyanjui, S. T. Norberg, C. S. Knee, I. Ahmed, S. Hull, L. Buannic, I. Hung, Z. Gan, F. Blanc, C. P. Grey, and S. G. Eriksson, J. Mater. Chem. A 4, 5088 (2016).
[29] S. Dabaghmanesh, R. Saniz, M. N. Amini, D. Lamoen, and B. Partoens, J. Phys.: Condens. Matter 25, 415503 (2013).

[30] D. O. Scanlon, Phys. Rev. B 87, 161201(R) (2013).

[31] W. Kohn and L. J. Sham, Phys. Rev. 140, A1133 (1965).

[32] X. Gonze, F. Jollet et al., Comput. Phys. Commun. 205, 106 (2016).

[33] M. Torrent, François Jollet, François Bottin, Gilles Zérah, and X. Gonze, Comput. Mater. Sci. 42, 337 (2008).

[34] J. P. Perdew, K. Burke, and M. Ernzerhof, Phys. Rev. Lett. 77, 3865 (1996).

[35] G. Makov and M. C. Payne, Phys. Rev. B 51, 4014 (1995).

[36] A. Zywietz, J. Furthmüller, and F. Bechstedt, Phys. Rev. B 59 , 15166 (1999).

[37] W. E., W. Ren, and E. Vanden-Eijnden, Phys. Rev. B 66, 052301 (2002).

[38] A. I. Liechtenstein, V. I. Anisimov, and J. Zaanen, Phys. Rev. B 52, R5467 (1995).

[39] O. Bengone, M. Alouani, P. Blöchl, and J. Hugel, Phys. Rev. B 62, 16392 (2000).

[40] B. Amadon, F. Jollet, and M. Torrent, Phys. Rev. B 77, 155104 (2008).

[41] D. D. O'Regan, N. D. M. Hine, M. C. Payne, and A. A. Mostofi, Phys. Rev. B 82, 081102 (2010).

[42] J. P. Perdew and A. Zunger, Phys. Rev. B 23, 5048 (1981).

[43] G. Jomard, B. Amadon, F. Bottin, and M. Torrent, Phys. Rev. B 78, 075125 (2008)

[44] B. Dorado, M. Freyss, B. Amadon, M. Bertolus, G. Jomard, and P. Garcia, J. Phys.: Condens. Matter 25, 333201 (2013).

[45] A. Kokalj, Comp. Mater. Sci. 28, 155 (2003).

[46] Freezing the $4 d$ electrons of $\mathrm{Sn}$ in the core of the atomic data set slightly changes these two values to $0.72 \mu_{B}$ and $0.05 \mu_{B}$.

[47] G. Geneste and G. Dezanneau, Solid State Ionics 308, 121 (2017).

[48] A. L. Schluger and A. M. Stoneham, J. Phys.: Condens. Matter 5, 3049 (1993).

[49] R. Hempelmann, Phys. B (Amsterdam) 226, 72 (1996); R. Hempelmann, Ch. Karmonik, Th. Matzke, M. Cappadonia, U. Stimming, T. Springer, and M. A. Adams, Solid State Ionics 77, 152 (1995).

[50] M. Björketun, P. G. Sundell, and G. Wahnström, Phys. Rev. B 76, 054307 (2007).

[51] F. Jollet, M. Torrent, and N. Holzwarth, Comput. Phys. Comm. 185, 1246 (2014).

[52] H. Park, A. J. Millis, and C. A. Marianetti, Phys. Rev. B 92, 035146 (2015).

[53] Y. C. Wang, Z. H. Chen, and H. Jiang, J. Chem. Phys. 144, 144106 (2016).

[54] G. Kresse and D. Joubert, Phys. Rev. B 59, 1758 (1999).

[55] Z. Wang, C. Brock, A. Matt, and K. H. Bevan, Phys. Rev. B 96, 125150 (2017). 